\title{
Site Specific Soil Fertility Ranking and Seasonal Paddy Variety Selection: An Intuitionistic Fuzzy Rough Set and Fuzzy Bayesian Based Decision Model
}

\author{
K. Lavanya, M. A. Saleem Durai and N. Ch. S. N. Iyengar \\ School of computing science and engineering, \\ VIT University, Vellore, Tamilnadu, India, \\ lavanya.sendhilvel@gmail.com,masaleemdurai@vit.ac.in,nchsniyengar48@gmail.com.
}

\begin{abstract}
In decision making, crisp ranking is not possible when the entire attribute characteristics and their degree of importance are known precisely. In real world situations decision making takes place in an environment where the goals, the constraints, and the consequences of possible actions are not known precisely. Thus the best condition for classic decision making problem may not be satisfied when the situation involves both fuzzy and crisp data. Site specific soil fertility and seasonal crop selection data are characterized by high degree of fuzziness and uncertainty. In our model, intuitionistic fuzzy rough set establishes a close connection between the concepts of similarity and dissimilarity thereby providing an excellent framework for ranking soil fertility. Further fuzzy Bayesian incorporates both fuzzy and uncertainty in the probability model yielding more realistic seasonal paddy variety selection. The decision model introduced in this paper is suitable for both data rich and data poor environment. The results illustrate that the soil fertility ranking and successive paddy variety selection can help to sustain the soil fertility in subsequent rotations and minimize the loss of nutrients from the sites.
\end{abstract}

Keywords: Fuzzy Bayesian, Uncertainty, Similarity, Dissimilarity, Ranking, Crop selection

\section{Introduction}

India has the largest paddy output in the world and is also the second largest exporter of rice in the world. Soil is the natural and vital resource for growing food, fiber and firewood to meet human needs. Declining soil fertility is a major constraint on crop production in the semi-arid highlands of Tamilnadu, India. One of the major constraints for crop production faced by smallholder farmers is the inadequate supply of nutrients [15]. Farmers either entirely discard the conventional practice of using natural fertilizer to reinstate soil fertility, or unable to put down land uncultivated for long enough for it to be effective. Reviewing the past, most research concentrated on trials to decide the suitable amount, type of fertilizer for best yields in specific soil types and agro-ecological locations. Here we concentrate in the civilizing farmer's knowledge and their experience as an essential element to build local systems of knowledge related to specific locations and understanding the local conditions of production. Then the identified soil samples are ranked based on intuitionistic fuzzy proximity relation and rough sets according to their fertility and potential productivity. Fuzzy theory provides a tool to describe the characteristics of a too complex or ill-defined system to admit precise mathematical analysis. Each element of the universe of discourse is indicated by the degree of belongingness to a set in the range [0-1]. The degree of non-belongingness is just automatically the complement to 1 of the membership degree. But, in real time there is chance of hesitation. Atanassov [1] introduced the concept of intuitionistic fuzzy set 
which is a natural generalization of fuzzy set, useful in modeling many real life situations. Each intuitionistic fuzzy number is interpreted as an ensemble of fuzzy numbers. This enables to define a fuzzy rank and characteristic vagueness factor for each intuitionistic fuzzy number. We presented intuitionistic fuzzy sets as a tool for reasoning in the presence of imperfect facts and imprecise knowledge. Diverse paddy varieties have unique needs for nutrients, light, water, temperature and air [21]. When either of these basic needs is not met, the crop will be stressed and will not grow properly. Selecting crop varieties is therefore a very important exercise that a farmer needs to undertake. Classical Bayesian method characterizes future state prediction as probability events. But the crop selection (fuzzy) data are vague, uncertain and ambiguous. The fusion of fuzzy sets with Bayesian enables to combine uncertainty handling with approximate reasoning. This enhances the representative component of Bayes inference using the knowledge inherent in fuzzy logic, leading to better robustness, noise immunity, and applicability in uncertain/imprecise contexts.

The structure of paper is organized as follows: Section 2 and 3 highlights the background study. Proposed System is presented in Section 4 and evaluation of the proposed system is discussed in Section 5.

\section{Related Works}

In this section, the various literature studies related to decision models for uncertain and vague information are reviewed. De Cock et al., [3] represented linguistic hedges (eg cool, warm) as fuzzy rough approximations using mutual similarity relations in the domain of discourse. H.Garg et al., [4] presented a hybridized technique named as particle swarm optimization based vague cut set for determining the membership and nonmembership function of fuzzy system reliability. To remove uncertainty the system uses ordinary arithmetic operations instead of fuzzy arithmetic operation and particle swarm optimization to construct membership functions. A.Zeng et al., [5] used principal component analysis based quantitative index to measure the relative importance of different conditions constructed by all condition attributes that strengthens the attribute and their value reductions for maintaining the decision table's discernibility relations. Z.S.Xu et al.,[7] developed the concepts of intuitionistic fuzzy weighted geometric operator, intuitionistic fuzzy ordered weighted geometric operator, and intuitionistic fuzzy hybrid geometric operator to accommodate the environment in which the given arguments are intuitionistic fuzzy sets characterized by a membership function and a nonmembership function. M.Kucukvar et al., [9] proposed a fuzzy multi-criteria decision making method for ranking the life cycle sustainability performance of different pavement alternatives constructed with hot-mix and warm-mix asphalt mixtures. It was tested in several life cycle phases such as raw material extraction and processing, manufacturing and end-of-life. Cesar et al., [10] presented an approach to predict compressor performance using Bayesian networks and a hybrid Fuzzy-Bayesian network. The proposed Fuzzy-Bayesian network structure achieved a significant time reduction to one third of the complete test time on average, in the refrigerating capacity evaluation. Liessman Sturlaugson et al.,[12] illustrated Bayesian networks (BNs) as a common datadriven approach for representing and reasoning in the presence of uncertainty. Bayesian is used as an intractable decider when the complexity of network increases with much number of nodes and states. N.Cagman et al.,[13] demonstrated fuzzy soft aggregation operator for constructing more efficient decision making method. The crisp parameter sets were approximated by fuzzy subsets of universe and the attribute with largest membership grade was represented as the decision value. According to Jiang Y et al., [14] the adjustable approach to fuzzy soft sets leads to dynamic decision making. The designed approach is specific to intuitionistic fuzzy soft by using level soft sets of intuitionistic fuzzy soft sets. Kukuvar et al.,[16] utilized intuitionistic fuzzy entropy method to identify 
the importance of phases and criteria, intuitionistic fuzzy weighted geometric averaging operator to establish a sub-decision making matrix based on weights of attribute, and intuitionistic fuzzy weighted arithmetic averaging operator to build a super decision matrix depending on weights of different life cycle phases. Chu et al.,[17] aimed to develop a method for solving pattern recognition problems under the Atanassov's intuitionistic fuzzy sets based on similarity measures. . In addition for the convenience of computing and ranking processes, a computer interface decision support system was also developed to help decision maker make diagnoses more efficiently. Shu-Ping et al.,[18] concentrated in multi attribute decision making problems whose uncertain attributes are expressed using triangular intuitionistic fuzzy numbers. It used the ratio of the possibility mean to the possibility standard deviation to solve possibility mean and variance of triangular intuitionistic fuzzy numbers. Polat.S et al.,[20] considered a priority ranking system for contaminated sites in which vagueness in parameter values are considered. It aims to evaluate potential human health risks due to contamination using sufficiently comprehensive and readily available parameters in describing the fate and transport of contaminants in air, soil, and groundwater. The Vagueness in these parameter values were solved by means of fuzzy set theory. Mueller et al.,[21] elaborated studies of soil structure importance and its preservation methods for overall soil quality assessment. According to their results soils with clay contents $>30 \%$ ie., unfavorable soil structure could not be reliably recognized for evaluation of visual soil structure. C.J. Pilbeam et al.,[22] used triangulated data to determine the existing practices for soil fertility management, the extent of such practices, and the perception of the direction of changes in soil fertility. The survey results showed that the two principal farmer practices for retaining soil fertility are the application of farmyard manure (FYM) and of chemical fertilizer (mainly urea and di ammonium phosphate).W.S. Lee et al., [24] made a comparative study of diverse types of sensors and instruments such as field-based electronic sensors, spectro radiometers, machine vision, airborne multispectral and hyperspectral remote sensing, satellite imagery, thermal imaging, RFID, and machine olfaction system. It also presented a review of these sensing technologies and discusses how they are used for precision agriculture and crop management, especially for specialty crops. From the related works it is evident that the imprecise and vague nature of information in decision making have large impact in aspects such as accuracy, reliability and time consumption.

\section{Background Study}

\subsection{Information Table}

Given $(U, A)$ be an information system where $\mathrm{U}$ be the finite non empty set (universe) of objects and $\mathrm{A}$ is non-empty finite set of attributes (features, variables).For every $a \varepsilon A$, $V_{a}$ is the set of values attribute 'a' may take, called domain of attribute A. In addition every attribute $a \varepsilon A$ defines an information function, $D_{a}: U \rightarrow V$.

Table1. Coded Information Table

\begin{tabular}{c|c|c|c}
\hline $\mathbf{U}$ & $\mathbf{a}_{1}$ & $\mathbf{a}_{2}$ & $\mathbf{D}$ \\
\hline $\mathrm{x}_{1}$ & large & 200 & Excellent \\
\hline $\mathrm{x}_{2}$ & large & 100 & Excellent \\
\hline $\mathrm{x}_{3}$ & large & 250 & Excellent \\
\hline $\mathrm{x}_{4}$ & medium & 200 & Average \\
\hline $\mathrm{x}_{5}$ & medium & 200 & Average \\
\hline $\mathrm{x}_{6}$ & medium & 100 & Excellent \\
\hline $\mathrm{x}_{7}$ & medium & 100 & Bad \\
\hline $\mathrm{x}_{8}$ & medium & 100 & Bad \\
\hline $\mathrm{x}_{9}$ & small & 200 & Average \\
\hline
\end{tabular}


\begin{tabular}{cc|c|c|c}
\hline $\mathrm{x}_{10}$ & small & 250 & Average \\
\hline In Table 1, $U=\left\{x_{1}, x_{2}, x_{3}, x_{4}, x_{5}, x_{6} \ldots \ldots \ldots \ldots x_{10}\right\} ; A=\left\{a_{1}, a_{2}, d\right\}$. The domains of attributes are
\end{tabular} $V_{1}\left(\right.$ for $\left.a_{1}\right)=\{$ large, medium $\} ; V_{2}\left(\right.$ for $\left.a_{2}\right)=\{100,200,250\} ; V_{3}($ for $d)=\{$ excellent, average, bad\}

\subsection{Ordered Information Table}

An information table does not consider any semantic relationships between distinct values of a particular attribute. By incorporating semantics information, we may obtain various generalizations of information tables. Ordered information tables may be viewed as information Tables with added semantics [1].

An ordered information table is a pair:

$O I T=\left\{I T, \mathrm{f}_{a} / a \varepsilon A\right\}$

Where IT is information table and $\mathrm{f}_{a}$ is weak order on $V_{a}$. An ordering of values of a particular attribute naturally induces ordering of objects namely $V_{a 1} \mathrm{f}_{a} V_{a 2}$ If and If only $f\left(V_{a 1}\right) \mathrm{f}_{a} f\left(V_{a 2}\right)$

An object $x_{i}$ is ranked ahead of object $x_{j}$ if and only if the value of $x_{i}$ on the attribute a is ranked ahead of the value of $x_{j}$ on the attribute $a$.Suppose for the information Table 1, the ordering can be defined as follows

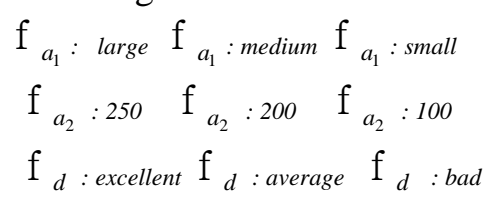

\subsection{Inutionistic Fuzzy Sets}

In Fuzzy set theory, the membership of every element to a fuzzy set is a single value between [0-1] .But in real cases there may be some hesitation cases. The concept of Intuitionist fuzzy gives the possibility to model unknown information with an additional degree. An intuitionistic fuzzy set is an object having the following form

$A=\left\{\left(\mu_{A}(a), \gamma_{A}(\mathrm{a}), \alpha_{A}(a)\right) / a \varepsilon A\right\}$

Where $\mu_{A}(a), \gamma_{A}(\mathrm{a}), \alpha_{A}(a)$ represent membership, non-membership and intermediate degree of a in A respectively with the condition

$$
\begin{aligned}
& 0 \leq \mu_{A}(a)+\gamma_{A}(\mathrm{a})+\alpha_{A}(a) \leq 1, \text { for all } a \varepsilon A \\
& \mu_{A}(a) \rightarrow[0,1], a \varepsilon A \rightarrow \mu_{A}(a) \varepsilon[0,1] \\
& \gamma_{A}(\mathrm{a}) \rightarrow[0,1], a \varepsilon A \rightarrow \gamma_{A}(\mathrm{a}) \varepsilon[0,1] \\
& \alpha_{A}(a) \rightarrow[0,1], a \varepsilon A \rightarrow \alpha_{A}(a) \varepsilon[0,1]
\end{aligned}
$$

\subsection{Intuitionistic Fuzzy Proximity Relation}

The concept of intuitionistic fuzzy proximity relation finds the attribute values that are $(\alpha, \beta)$ identical before introducing the feature ranking $(\alpha=\operatorname{similarity} ; \beta=$ dissimilarity $)$ [8]. This is because feature extraction is not possible when the information gain of each attribute is not known. In discernibility matrix of Table 1 if value of $\alpha=1, \beta=0$, then the relation reduces to indiscernibility relation

\subsection{Rough Set}

Rough set is a formal approximation of a crisp set (i.e., conventional set) in terms of a pair of sets which give the lower and the upper approximation of the original set [2]. It analyzes attributes with real values and categorizes them into intervals. 


\subsection{Indiscernibility Relation}

Two objects $x_{i}, x_{j}$ are said to be indiscernible by their set of attributes a where $a \varepsilon A$, if $a\left(x_{i}\right)=a\left(x_{j}\right)$ i.e., every element in the subset a must be equal. It is generally represented as $\operatorname{Ind}(\mathrm{A})$

\subsection{Lower and Upper Approximations}

For data analysis rough set approach defines two basic concepts namely the lower and the upper approximations of a set. The lower approximation of the set $\mathrm{X}$ is a set of objects $\mathrm{x}_{\mathrm{i}}$, belonging to the elementary sets contained in $\mathrm{X}$ (of space $\mathrm{R}$ ). The upper approximation is the union of elementary sets with a non empty intersection to $\mathrm{X}$.

$$
\begin{aligned}
& \underline{R} X=\bigcup\{Y \in U / R: Y \subseteq X\} \\
& \bar{R} X=\bigcup\{Y \in U / R: Y \cap X \neq \phi\}
\end{aligned}
$$

The $R$-boundary of $X, B N_{R}(X)$ is given by $B N_{R}(X)=\bar{R} X-\underline{R} X$. We say $X$ is rough with respect to $R$ if and only if $\bar{R} X \neq \underline{R} X$, equivalently $B N_{R}(\mathrm{X}) \neq \phi . X$ is said to be $R-$ definable if and only if $\bar{R} X=\underline{R} X$ or $B N_{R}(X)=\phi$. So, a set is rough with respect to $R$ if and only if it is not $R$-definable

\section{Architecture}

Decision dependency has become a common form of knowledge representation owing to its properties of expressiveness and ease of understanding. A real time decision Table includes a vector of decision and conditional attributes that describe the objectives and constraints. These attributes describe the characteristics, qualities or performance parameters of alternatives. The real time decision making makes the choice of reasonable and justifiable from divisive alternatives [13]. The existence of fuzzy and crisp data has a high degree of uncertainty that leads to inappropriate conclusions due to differences in priorities and objectives. The application of rough intuitionistic fuzzy concepts to ranking of decision dependencies and fuzzy Bayesian for selection of particular object from a decision table results in non-redundant set of decisions.

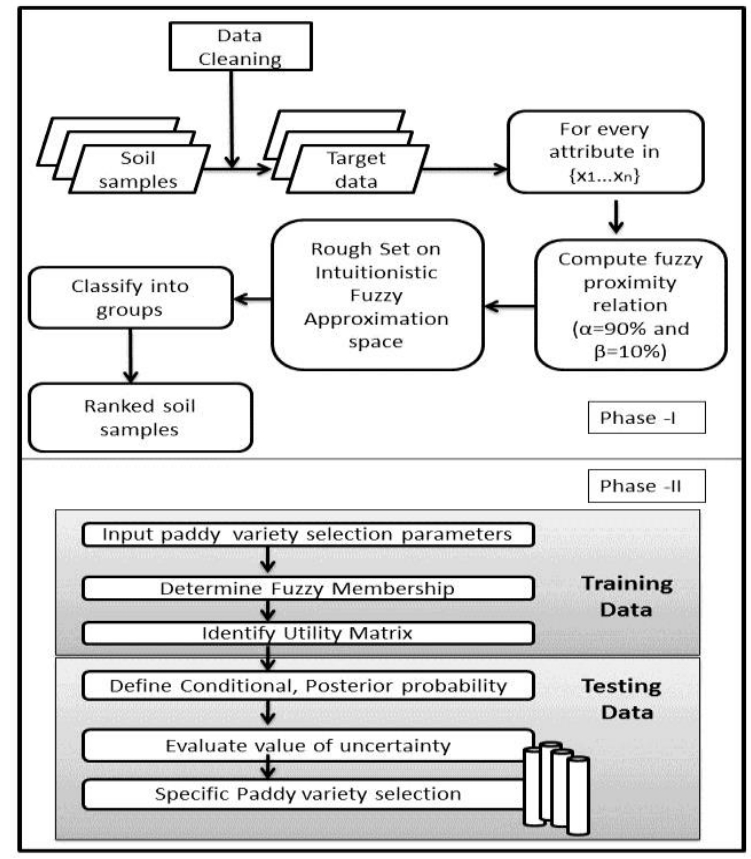

Figure 1. Proposed Architecture 


\section{Phase-I}

It involves in the generation of soil ranking to determine fertility with respect to the number of data set.

\section{Phase-II}

It applies combined fuzzy and Bayesian methodology on the crop selection parameters to decide appropriate paddy variety for cultivation.

\subsection{Data Description}

This study was carried out in Ariyur village of Kaveripakkam block, Vellore district, Tamilnadu, India. The major crops cultivated are paddy and sugarcane. The predominant agricultural practice is small-scale mixed subsistence farming. Average landholdings in the village are less than one hectare. A total of 100 soil samples were collected from farmers of various age groups. The collected samples were analyzed in the district soil test laboratory (STL) of Department of Agriculture. The main soil types identified were red sandy loam, clay loam and saline. $20 \%$ of soil samples were used for ranking soil fertility and $80 \%$ were used for specific paddy variety selection.

\subsection{Intuitionistic Fuzzy Rough Set based Soil Fertility Ranking}

Rough set theory is used to study mixed types of data such as continuous, valued and symbolic data [3].It is a generalization of crisp rough set to deal with data sets of real time attributes. Intuitionistic Fuzzy Rough set employs a fuzzy similarity relation to characterize the similarity degree between two objects thereby encapsulates the distinct concepts of vagueness and indiscernibility in uncertain data (Algorithm I)

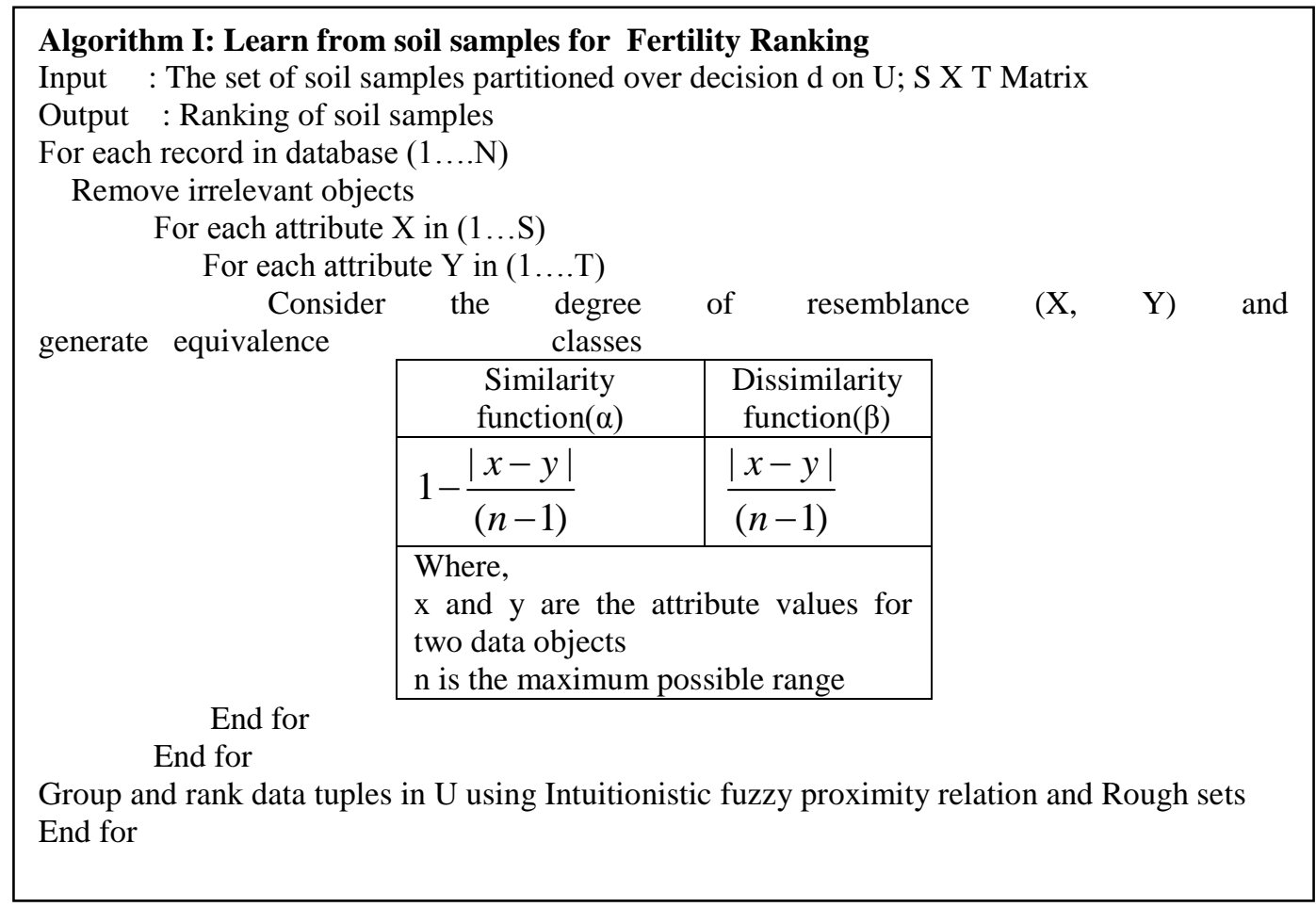

Soil is a major source of three main nutrients namely nitrogen $(\mathrm{N})$, phosphorus $(\mathrm{P})$ and potassium $(\mathrm{K})$. Together they make up the trio known as NPK. Other important nutrients are calcium, magnesium and sulfur. Plants also need small quantities of iron, manganese, zinc and copper, known as trace elements. It is necessary to identify soil fertility so that essential nutrient supplement can be identified at proper stage and also to minimize the 
usage of unhealthy inorganic chemical fertilizers thereby the financial burden. The major attributes that play a vital role and the notations that are used in our analysis is given in Table 2

Table 2. Notation Representation

\begin{tabular}{l|l|l}
\hline Attribute & Notation & Possible Range \\
\hline $\begin{array}{l}\text { Electrical } \\
\text { Conductivity }\end{array}$ & $\mathrm{Ec}$ & $1-3$ \\
\hline Acidity/Alkalinity & $\mathrm{pH}$ & $1-7.5$ \\
\hline Nitrogen & $\mathrm{N}$ & $\begin{array}{l}1-450 \\
\mathrm{~kg} / \mathrm{hectare}\end{array}$ \\
\hline Phosphorous & $\mathrm{P}$ & $\begin{array}{l}1-22 \\
\mathrm{~kg} / \mathrm{hectare}\end{array}$ \\
\hline Potassium & $\mathrm{K}$ & $\begin{array}{l}1-280 \\
\mathrm{~kg} / \mathrm{hectare}\end{array}$ \\
\hline Zinc & & $1-3 \mathrm{ppm}$ \\
\hline Copper & $\mathrm{Zn}$ & $1-3 \mathrm{ppm}$ \\
\hline Iron & $\mathrm{Cu}$ & $1-8 \mathrm{ppm}$ \\
\hline Manganese & $\mathrm{Fe}$ & $1-4 \mathrm{ppm}$ \\
\hline
\end{tabular}

Table 3 represents the nutrient status of soil samples (20\%) collected from Ariyur village. It forms as target data set where the attributes are defined based on the problem objective. Literature and numerical values for soil fertility factors were collected and studied. The nutrient values forms the attribute set for our analysis. The soil fertility ranking becomes our decision variable. Columns of the table are labeled by nutrients (attributes) and rows by soil samples (objects), whereas each cell of the Table is attribute values. Therefore, each row of the Table can be seen as information about specific farmer field.

Table 3. Nutrition Status of Soil Samples

\begin{tabular}{l|l|l|l|l|l|l|l|l|l}
\hline Samples & $\mathbf{E C}$ & $\mathbf{P H}$ & $\mathbf{N}$ & $\mathbf{P}$ & $\mathbf{K}$ & $\mathbf{Z n}$ & $\mathbf{C u}$ & $\mathbf{F e}$ & $\mathbf{M n}$ \\
\hline $\mathrm{x}_{1}$ & 0.24 & 7.3 & 113 & 11.5 & 96 & 0.5 & 2.2 & 7.5 & 3.3 \\
\hline $\mathrm{x}_{2}$ & 0.25 & 7.2 & 65 & 126 & 92 & 0.5 & 2.6 & 7.3 & 3.6 \\
\hline $\mathrm{x}_{3}$ & 0.25 & 7.3 & 135 & 9.3 & 96 & 0.60 & 1.60 & 3.60 & 2.30 \\
\hline $\mathrm{x}_{4}$ & 0.23 & 7.3 & 131 & 4.9 & 88 & 0.60 & 1.30 & 3.30 & 2.50 \\
\hline $\mathrm{x}_{5}$ & 0.21 & 7.6 & 195 & 8.2 & 84 & 0.50 & 1.20 & 3.20 & 2.40 \\
\hline $\mathrm{x}_{6}$ & 0.26 & 7.5 & 116 & 9.3 & 92 & 0.50 & 1.10 & 3.10 & 2.00 \\
\hline $\mathrm{x}_{7}$ & 0.21 & 7.5 & 120 & 10.4 & 84 & 0.50 & 1.00 & 4.00 & 2.10 \\
\hline $\mathrm{x}_{8}$ & 0.24 & 7.5 & 105 & 11.5 & 129 & 0.60 & 1.40 & 4.40 & 2.20 \\
\hline $\mathrm{x}_{9}$ & 0.26 & 7.4 & 120 & 12.6 & 88 & 0.70 & 2.50 & 4.50 & 2.30 \\
\hline $\mathrm{x}_{10}$ & 0.24 & 7.4 & 143 & 10.4 & 111 & 0.70 & 2.30 & 3.30 & 2.50 \\
\hline $\mathrm{x}_{11}$ & 0.25 & 7.5 & 131 & 9.3 & 96 & 0.80 & 2.20 & 3.60 & 2.40 \\
\hline $\mathrm{x}_{12}$ & 0.35 & 7.3 & 210 & 8.2 & 88 & 0.80 & 2.60 & 3.30 & 2.60 \\
\hline $\mathrm{x}_{13}$ & 0.34 & 7.4 & 120 & 11.5 & 100 & 0.70 & 2.10 & 3.20 & 2.10 \\
\hline $\mathrm{x}_{14}$ & 0.26 & 7.5 & 168 & 9.3 & 92 & 0.90 & 2.70 & 3.10 & 2.60 \\
\hline $\mathrm{x}_{15}$ & 0.36 & 7.6 & 135 & 8.2 & 88 & 0.90 & 2.80 & 3.00 & 2.20 \\
\hline $\mathrm{x}_{16}$ & 0.24 & 7.3 & 120 & 10.4 & 96 & 0.50 & 2.30 & 3.40 & 2.30 \\
\hline $\mathrm{x}_{17}$ & 0.29 & 7.2 & 105 & 11.5 & 100 & 0.60 & 2.40 & 3.50 & 2.50 \\
\hline
\end{tabular}




\begin{tabular}{l|l|l|l|l|l|l|l|l|l}
\hline $\mathrm{x}_{18}$ & 0.26 & 7.7 & 153 & 7.1 & 150 & 0.60 & 1.30 & 3.30 & 2.40 \\
\hline $\mathrm{x}_{19}$ & 0.37 & 7.3 & 210 & 10.4 & 169 & 0.60 & 1.20 & 3.10 & 2.10 \\
\hline $\mathrm{x}_{20}$ & 0.28 & 7.4 & 172.5 & 11.5 & 169 & 0.60 & 1.10 & 4.00 & 2.20 \\
\hline
\end{tabular}

We aim to rank soil fertility using the fuzzy proximity relation so as to decide the amount of fertilizer usage with the help of domain expertise knowledge. Using the algorithm for soil fertility ranking, the intuitionistic fuzzy proximity relations is applied for the samples $\left\{x_{1}, x_{2} \ldots \ldots x_{20}\right\}$ to calculate approximate similarity and dissimilarity between them. Considering the degree of resemblance measures $\alpha>0.90$ and $\beta<0.10$ (similarity and dissimilarity) in nutrition status values, the obtained equivalence classes are represented in Table 4. Due to limitation of pages the calculation for $\mathrm{pH}, \mathrm{N}, \mathrm{P}, \mathrm{K}, \mathrm{Zn}, \mathrm{Cu}$ and $\mathrm{Fe}$ are omitted.

Table 4.1 Intuitionistic Fuzzy Proximity Relation of Ec

\begin{tabular}{|c|c|c|c|c|c|c|c|c|c|c|c|c|c|c|c|c|c|c|c|c|}
\hline EC & $\mathbf{x}_{1}$ & $\mathrm{x}_{2}$ & $x_{3}$ & $\mathbf{x}_{4}$ & $\mathbf{x}_{5}$ & $\mathrm{x}_{6}$ & $\mathrm{x}_{7}$ & $\mathrm{x}_{8}$ & $\mathbf{x}_{9}$ & $\begin{array}{l}x_{10} \\
\text { nat }\end{array}$ & $\mathbf{x}_{11}$ & $x_{12}$ & $\mathbf{x}_{13}$ & $\begin{array}{l}X_{14} \\
\text { nat }\end{array}$ & $\mathrm{X}_{15}$ & $x_{16}$ & $\begin{array}{l}\mathbf{x}_{17} \\
\end{array}$ & $\mathbf{X}_{18}$ & \begin{tabular}{|l|}
$X_{19}$ \\
na
\end{tabular} & $\mathbf{x}_{20}$ \\
\hline \multirow[t]{2}{*}{$\mathbf{x}_{1}$} & 1.0 , & 0.95 & 0.95 & 0.95 & 0.95 & 0.95 & 0.95 & 0.95 & 0.95 & 0.95 & 0.95 & 0.95 & 0.95 & 0.95 & 0.95 & 0.95 & 0.95 & 0.95 & 0.95 & 0.95 \\
\hline & 0.0 & 0.05 & 0.05 & 0.05 & 0.05 & 0.05 & 0.05 & 0.05 & 0.05 & 0.05 & 0.05 & 0.05 & 0.05 & 0.05 & 0.05 & 0.05 & 0.05 & 0.05 & 0.05 & 0.05 \\
\hline \multirow[t]{2}{*}{$\mathbf{x}_{2}$} & 0.95 & 1.0, & 0.97 , & 0.97 , & 0.97 , & 0.97 & 0.97 & 0.97 & 0.97 , & 0.97 , & 0.97 & 0.97 , & 0.97 , & 0.97 & 0.97 & 0.97 & 0.97 & 0.97 & 0.97 & 0.97 , \\
\hline & 0.05 & 0.0 & 0.009 & 0.009 & 0.009 & 0.009 & 0.009 & 0.009 & 0.009 & 0.009 & 0.009 & 0.009 & 0.009 & 0.009 & 0.009 & 0.009 & 0.009 & 0.009 & 0.009 & 0.009 \\
\hline \multirow[t]{2}{*}{$x_{3}$} & 0.99 & 0.97 & 1.0 & 0.99 & 0.99 & 0.99 & 0.99 & 0.99 & 0.99 & 0.99 & 0.99 & 0.99 & 0.99 & 0.99 & 0.99, & 0.99 & 0.99 & 0.99 & 0.99 & 0.99 , \\
\hline & 0.006 & 0.009 & 0.0 & 0.014 & 0.014 & 0.014 & 0.014 & 0.014 & 0.014 & 0.014 & 0.014 & 0.014 & 0.014 & 0.014 & 0.014 & 0.014 & 0.014 & 0.014 & 0.014 & 0.014 \\
\hline \multirow[t]{2}{*}{$x_{4}$} & 0.99 & 0.94 & 0.99 , & 1.0 & 0.99 , & 0.99 & 0.99 & 0.99 & 0.99 , & 0.99 , & 0.99 & 0.99 , & 0.99 & 0.99 & 0.99 & 0.99 & 0.99 & 0.99 & 0.99 & 0.99 , \\
\hline & 0.006 & 0.06 & 0.014 & 0.0 & 0.007 & 0.007 & 0.007 & 0.007 & 0.007 & 0.007 & 0.007 & 0.007 & 0.007 & 0.007 & 0.007 & 0.007 & 0.007 & 0.007 & 0.007 & 0.007 \\
\hline \multirow[t]{2}{*}{$\mathbf{x}_{5}$} & 1.0 , & 0.96 & 0.95 & 0.99 , & 1.0, & 0.96 & 0.96 & 0.96 & 0.96 & 0.96 & 0.96 & 0.96 & 0.96 & 0.96 & 0.96 & 0.96 & 0.96 & 0.96 & 0.96 & 0.96 , \\
\hline & 0.003 & 0.04 & 0.05 & 0.007 & 0.0 & 0.036 & 0.036 & 0.036 & 0.036 & 0.036 & 0.036 & 0.036 & 0.036 & 0.036 & 0.036 & 0.036 & 0.036 & 0.036 & 0.036 & 0.036 \\
\hline \multirow[t]{2}{*}{$\mathbf{x}_{6}$} & 0.99 & 0.91, & 0.98 & 0.97 , & 0.96 & 1.0 & 0.97 & 0.97, & 0.97 , & 0.97 , & $\begin{array}{l}0.97 \\
\end{array}$ & 0.97 , & 0.97 & 0.97 & 0.97 & 0.97 & 0.97, & 0.97 & 0.97 & 0.97 , \\
\hline & 0.004 & 0.09 & 0.021 & 0.027 & 0.036 & 0.0 & 0.03 & 0.03 & 0.03 & 0.03 & 0.03 & 0.03 & 0.03 & 0.03 & 0.03 & 0.03 & 0.03 & 0.03 & 0.03 & 0.03 \\
\hline \multirow[t]{2}{*}{$x_{7}$} & 0.99, & 0.94 & 0.97 , & 1.0 & 0.99 , & 0.97 & 1.0, & 1.0, & 1.0 & 1.0 & 1.0 & 1.0 & 1.0 & 1.0 & 1.0 & 1.0 & 1.0 & 1.0, & 1.0, & 1.0 \\
\hline & 0.014 & 0.06 & 0.031 & 0.0 & 0.01 & 0.03 & 0.0 & 0.002 & 0.002 & 0.002 & 0.002 & 0.002 & 0.002 & 0.002 & 0.002 & 0.002 & 0.002 & 0.002 & 0.002 & 0.002 \\
\hline \multirow[t]{2}{*}{$\mathbf{x}_{8}$} & 0.99, & 0.94 & 0.96 & 1.0 & 0.98 & 0.98 & 1.0, & 1.0, & 0.99 & 0.99 , & 0.99 & 0.99 , & 0.99 & 0.99 & 0.99 & 0.99 & 0.99 & 0.99 & 0.99, & 0.99 , \\
\hline & 0.006 & 0.06 & 0.036 & 0.002 & 0.02 & 0.02 & 0.002 & 0.0 & 0.007 & 0.007 & 0.007 & 0.007 & 0.007 & 0.007 & 0.007 & 0.007 & 0.007 & 0.007 & 0.007 & 0.007 \\
\hline \multirow[t]{2}{*}{$x_{9}$} & 0.98 & 0.94 & 0.98 & 0.99 , & 0.97 & 0.99 & 0.99 & 0.99 & 1.0 & 0.98 & 0.98 & 0.98 & 0.98 & 0.98 & 0.98 & 0.98 & 0.98 & 0.98 & 0.98 & 0.98 \\
\hline & 0.02 & 0.06 & 0.02 & 0.009 & 0.03 & 0.011 & 0.009 & 0.007 & 0.0 & 0.018 & 0.018 & 0.018 & 0.018 & 0.018 & 0.018 & 0.018 & 0.018 & 0.018 & 0.018 & 0.018 \\
\hline \multirow[t]{2}{*}{$\mathbf{x}_{10}$} & 1.0, & 0.92 & 0.97 , & 1.0 & 0.99 , & 0.97 , & 1.0 & 1.0, & 0.98 & 1.0, & 0.99, & 0.99 , & 0.99 & 0.99 & 0.99 & 0.99 , & 0.99 & 0.99 & 0.99 & 0.99 , \\
\hline & 0.003 & 0.08 & 0.03 & 0.002 & 0.01 & 0.03 & 0.002 & 0.003 & 0.018 & 0.0 & 0.005 & 0.005 & 0.005 & 0.005 & 0.005 & 0.005 & 0.005 & 0.005 & 0.005 & 0.005 \\
\hline \multirow[t]{2}{*}{$\mathbf{x}_{11}$} & 0.99 & 0.93 & 0.96 & 1.0 & 1.0 & 0.98 & 1.0 , & 1.0 & 0.99 , & 0.99 & 1.0 , & 0.99 & 0.99 & 0.99 & 0.99 & 0.99 & 0.99 & 0.99 & 0.99, & 0.99 , \\
\hline & 0.007 & 0.07 & 0.04 & 0.003 & 0.002 & 0.011 & 0.003 & 0.002 & 0.006 & 0.005 & 0.0 & 0.008 & 0.008 & 0.008 & 0.008 & 0.008 & 0.008 & 0.008 & 0.008 & 0.008 \\
\hline \multirow[t]{2}{*}{$\begin{array}{l}\mathbf{x}_{12} \\
\end{array}$} & 0.97 & 0.92 & 1.0 & 0.98 & 1.0 & 0.99 & 0.98 & 0.99 & 1.0 & 0.98 & 0.99 & 1.0, & 0.95 & 0.95 & 0.95 & 0.95 & 0.95 & 0.95 & 0.95 & 0.95 \\
\hline & 0.03 & 0.08 & 0.002 & 0.02 & 0.003 & 0.01 & 0.016 & 0.009 & 0.002 & 0.02 & 0.008 & 0.0 & 0.04 & 0.04 & 0.04 & 0.04 & 0.04 & 0.04 & 0.04 & 0.04 \\
\hline \multirow[t]{2}{*}{$\begin{array}{l}x_{13} \\
\end{array}$} & 0.98 , & 0.97 & 0.98 & 0.97, & 0.99 , & 0.94 & 0.97, & 0.97, & 0.95 & 0.97 , & 0.96 & 0.95 & 1.0 & 0.98 & 0.98 & 0.98 & 0.98 & 0.98 & 0.98 & 0.98 \\
\hline & 0.018 & 0.026 & 0.02 & 0.025 & 0.01 & 0.06 & 0.031 & 0.03 & 0.05 & 0.03 & 0.04 & 0.04 & 0.0 & 0.019 & 0.019 & 0.019 & 0.019 & 0.019 & 0.019 & 0.019 \\
\hline \multirow[t]{2}{*}{\begin{tabular}{|l}
$x_{14}$ \\
.
\end{tabular}} & 1.0 , & 0.95 & 0.98 & 1.0 & 0.99 & 0.97 & 1.0 & 0.99 & 0.98 & 1.0 & 00.99, & 0.98 & 0.98 & 1.0 , & 1.0 , & 1.0 , & 1.0 , & 1.0 , & 1.0 , & 1.0 \\
\hline & 0.002 & 0.05 & 0.02 & 0.003 & 0.01 & 0.026 & 0.003 & 0.005 & 0.02 & 0.002 & 0.006 & 0.02 & 0.019 & 0.0 & 0.002 & 0.002 & 0.002 & 0.002 & 0.002 & 0.002 \\
\hline
\end{tabular}




\begin{tabular}{|c|c|c|c|c|c|c|c|c|c|c|c|c|c|c|c|c|c|c|c|c|}
\hline $\mathbf{x}_{15}$ & $\begin{array}{l}1.0, \\
0.003\end{array}$ & $\begin{array}{l}0.93, \\
0.07\end{array}$ & $\begin{array}{l}0.97, \\
0.025\end{array}$ & $\begin{array}{l}1.0, \\
0.002\end{array}$ & $\begin{array}{l}0.99 \\
0.01\end{array}$ & $\begin{array}{l}0.97, \\
0.03\end{array}$ & $\begin{array}{l}1.0, \\
0.003\end{array}$ & $\begin{array}{l}1.0, \\
0.003\end{array}$ & $\begin{array}{l}0.98 \\
0.016\end{array}$ & $\begin{array}{l}1.0, \\
0.0\end{array}$ & $\begin{array}{l}0.99 \\
0.005\end{array}$ & $\begin{array}{l}0.98 \\
0.018\end{array}$ & $\begin{array}{l}0.97 \\
0.027\end{array}$ & $\begin{array}{l}1.0, \\
0.002\end{array}$ & $\begin{array}{l}1.0, \\
0.0\end{array}$ & $\begin{array}{l}0.99, \\
0.005\end{array}$ & $\begin{array}{l}0.99, \\
0.005\end{array}$ & $\begin{array}{l}0.99, \\
0.005\end{array}$ & $\begin{array}{l}0.99 \\
0.005\end{array}$ & $\begin{array}{l}0.99, \\
0.005\end{array}$ \\
\hline$\overline{x_{16}}$ & $\begin{array}{l}0.99, \\
0.007\end{array}$ & $\begin{array}{l}0.93 \\
0.07\end{array}$ & $\begin{array}{l}1.0, \\
0.001\end{array}$ & $\begin{array}{l}1.0, \\
0.003\end{array}$ & $\begin{array}{l}0.98, \\
0.02\end{array}$ & $\begin{array}{l}0.98, \\
0.018\end{array}$ & $\begin{array}{l}1.0, \\
0.02\end{array}$ & $\begin{array}{l}1.0, \\
0.002\end{array}$ & $\begin{array}{l}0.99 \\
0.006\end{array}$ & $\begin{array}{l}0.99, \\
0.005\end{array}$ & $\begin{array}{l}1.0, \\
0.0\end{array}$ & $\begin{array}{l}0.99, \\
0.008\end{array}$ & $\begin{array}{l}0.96, \\
0.044\end{array}$ & $\begin{array}{l}0.99, \\
0.006\end{array}$ & $\begin{array}{l}0.99, \\
0.005\end{array}$ & $\begin{array}{l}1.0, \\
0.0\end{array}$ & $\begin{array}{l}0.97, \\
0.031\end{array}$ & $\begin{array}{l}0.97, \\
0.031\end{array}$ & $\begin{array}{l}0.97, \\
0.031\end{array}$ & $\begin{array}{l}0.97, \\
0.031\end{array}$ \\
\hline $\mathbf{x}_{17}$ & $\begin{array}{l}0.99, \\
0.005\end{array}$ & $\begin{array}{l}0.96, \\
0.036\end{array}$ & $\begin{array}{l}0.99, \\
0.014\end{array}$ & $\begin{array}{l}0.98 \\
0.02\end{array}$ & $\begin{array}{l}1.0, \\
0.003\end{array}$ & $\begin{array}{l}0.95 \\
0.046\end{array}$ & $\begin{array}{l}0.98 \\
0.02\end{array}$ & $\begin{array}{l}0.98, \\
0.016\end{array}$ & $\begin{array}{l}0.96 \\
0.04\end{array}$ & $\begin{array}{l}0.98, \\
0.017\end{array}$ & $\begin{array}{l}0.97 \\
0.027\end{array}$ & $\begin{array}{l}0.96 \\
0.038\end{array}$ & $\begin{array}{l}0.99 \\
0.013\end{array}$ & $\begin{array}{l}0.99 \\
0.006\end{array}$ & $\begin{array}{l}0.98, \\
0.017\end{array}$ & $\begin{array}{l}0.97, \\
0.031\end{array}$ & $\begin{array}{l}1.0, \\
0.0\end{array}$ & $\begin{array}{l}0.99, \\
0.014\end{array}$ & $\begin{array}{l}0.99 \\
0.014\end{array}$ & $\begin{array}{l}0.99, \\
0.014\end{array}$ \\
\hline $\mathrm{x}_{18}$ & $\begin{array}{l}1.0, \\
0.002\end{array}$ & $\begin{array}{l}0.95, \\
0.052\end{array}$ & $\begin{array}{l}0.98 \\
0.017\end{array}$ & $\begin{array}{l}0.99, \\
0.011\end{array}$ & $\begin{array}{l}1.0, \\
0.002\end{array}$ & $\begin{array}{l}0.96 \\
0.04\end{array}$ & $\begin{array}{l}0.99 \\
0.005\end{array}$ & $\begin{array}{l}0.99, \\
0.007\end{array}$ & $\begin{array}{l}0.97, \\
0.03\end{array}$ & $\begin{array}{l}0.99, \\
0.014\end{array}$ & $\begin{array}{l}0.98 \\
0.02\end{array}$ & $\begin{array}{l}0.97, \\
0.025\end{array}$ & $\begin{array}{l}0.98, \\
0.017\end{array}$ & $\begin{array}{l}1.0, \\
0.003\end{array}$ & $\begin{array}{l}0.99, \\
0.014\end{array}$ & $\begin{array}{l}0.98, \\
0.018\end{array}$ & $\begin{array}{l}0.99, \\
0.014\end{array}$ & $\begin{array}{l}1.0, \\
0.0\end{array}$ & $\begin{array}{l}1.0, \\
0.003\end{array}$ & $\begin{array}{l}1.0, \\
0.003\end{array}$ \\
\hline$\overline{x_{19}}$ & $\begin{array}{l}1.0, \\
0.002\end{array}$ & $\begin{array}{l}1.0, \\
0.002\end{array}$ & $\begin{array}{l}1.0, \\
0.001\end{array}$ & $\begin{array}{l}1.0, \\
0.003\end{array}$ & $\begin{array}{l}0.99, \\
0.014\end{array}$ & $\begin{array}{l}0.97, \\
0.03\end{array}$ & $\begin{array}{l}1.0, \\
0.003\end{array}$ & $\begin{array}{l}0.99, \\
0.005\end{array}$ & $\begin{array}{l}0.98 \\
0.016\end{array}$ & $\begin{array}{l}1.0, \\
0.002\end{array}$ & $\begin{array}{l}0.99, \\
0.006\end{array}$ & $\begin{array}{l}0.98, \\
0.015\end{array}$ & $\begin{array}{l}0.98, \\
0.019\end{array}$ & $\begin{array}{l}.0, \\
0.0\end{array}$ & $\begin{array}{l}1.0, \\
0.002\end{array}$ & $\begin{array}{l}0.99, \\
0.01\end{array}$ & $\begin{array}{l}0.99, \\
0.006\end{array}$ & $\begin{array}{l}1.0, \\
0.003\end{array}$ & $\begin{array}{l}1.0, \\
0.0\end{array}$ & $\begin{array}{l}0.99, \\
0.006\end{array}$ \\
\hline $\mathbf{x}_{20}$ & $\begin{array}{l}0.99, \\
0.005\end{array}$ & $\begin{array}{l}0.96, \\
0.04\end{array}$ & $\begin{array}{l}0.99, \\
0.014\end{array}$ & $\begin{array}{l}0.98 \\
0.019\end{array}$ & $\begin{array}{l}1.0, \\
0.003\end{array}$ & $\begin{array}{l}0.95 \\
0.05\end{array}$ & 0.98 & $\begin{array}{l}0.98, \\
0.017\end{array}$ & $\begin{array}{l}0.96 \\
0.04\end{array}$ & $\begin{array}{l}0.98, \\
0.017\end{array}$ & $\begin{array}{l}0.97, \\
0.027\end{array}$ & $\begin{array}{l}0.96, \\
0.038\end{array}$ & $\begin{array}{l}0.99, \\
0.003\end{array}$ & $\begin{array}{l}0.99, \\
0.006\end{array}$ & $\begin{array}{l}0.98, \\
0.017\end{array}$ & $\begin{array}{l}0.97, \\
0.03\end{array}$ & $\begin{array}{l}1.0, \\
0.0\end{array}$ & $\begin{array}{l}0.99, \\
0.005\end{array}$ & $\begin{array}{l}0.99 \\
0.006\end{array}$ & $\begin{array}{l}1.0, \\
0.0\end{array}$ \\
\hline
\end{tabular}

Table 4.2 Intuitionistic Fuzzy Proximity Relation of Mn

\begin{tabular}{|c|c|c|c|c|c|c|c|c|c|c|c|c|c|c|c|c|c|c|c|c|}
\hline Mn & $\mathbf{x}_{1}$ & $\mathrm{x}_{2}$ & $\mathrm{x}_{3}$ & $\mathbf{x}_{4}$ & $\mathrm{x}_{5}$ & $\overline{\mathbf{x}_{6}}$ & $\begin{array}{l}\mathbf{x}_{7} \\
\end{array}$ & $\mathrm{x}_{\mathrm{s}}$ & $\mathbf{x}_{9}$ & $x_{10}$ & $x_{11}$ & $\mathbf{x}_{12}$ & $\mathbf{x}_{13}$ & $\mathbf{x}_{14}$ & $\mathrm{X}_{15}$ & $\mathbf{x}_{16}$ & $\mathbf{x}_{17}$ & $x_{18}$ & $x_{19}$ & $\mathbf{x}_{20}$ \\
\hline $\mathbf{x}_{1}$ & $\begin{array}{l}1.0, \\
0.0\end{array}$ & $\begin{array}{l}0.98, \\
0.021\end{array}$ & $\begin{array}{l}0.98, \\
0.02\end{array}$ & $\begin{array}{l}0.03, \\
0.97\end{array}$ & $\begin{array}{l}0.73, \\
0.27\end{array}$ & $\begin{array}{l}0.08, \\
0.916\end{array}$ & $\begin{array}{l}0.93, \\
0.07\end{array}$ & $\begin{array}{l}0.41, \\
0.59\end{array}$ & $\begin{array}{l}0.91, \\
0.087\end{array}$ & $\begin{array}{l}0.88, \\
0.22\end{array}$ & $\begin{array}{l}0.84, \\
0.16\end{array}$ & $\begin{array}{l}0.85, \\
0.153\end{array}$ & $\begin{array}{l}0.98, \\
0.018\end{array}$ & $\begin{array}{l}0.78, \\
0.22\end{array}$ & $\begin{array}{l}1.0, \\
0.0\end{array}$ & $\begin{array}{l}0.81, \\
0.19\end{array}$ & $\begin{array}{l}0.89, \\
0.11\end{array}$ & $\begin{array}{l}0.81, \\
0.192\end{array}$ & $\begin{array}{l}0.93, \\
0.068\end{array}$ & $\begin{array}{l}0.98, \\
0.02\end{array}$ \\
\hline $\mathrm{x}_{2}$ & $\begin{array}{l}0.98 \\
0.021\end{array}$ & $\begin{array}{l}1.0, \\
0.0\end{array}$ & $\begin{array}{l}1.0, \\
0.0\end{array}$ & $\begin{array}{l}1.0, \\
0.02\end{array}$ & $\begin{array}{l}0.97, \\
0.009\end{array}$ & $\begin{array}{l}0.95, \\
0.052\end{array}$ & $\begin{array}{l}0.81, \\
0.19\end{array}$ & $\begin{array}{l}0.88, \\
0.12\end{array}$ & $\begin{array}{l}0.93 \\
0.07\end{array}$ & $\begin{array}{l}0.78 \\
0.22\end{array}$ & $\begin{array}{l}0.83, \\
0.17\end{array}$ & $\begin{array}{l}0.86, \\
0.14\end{array}$ & $\begin{array}{l}1.0, \\
0.0\end{array}$ & $\begin{array}{l}0.95, \\
0.047\end{array}$ & $\begin{array}{l}0.98, \\
0.016\end{array}$ & $\begin{array}{l}0.88, \\
0.12\end{array}$ & $\begin{array}{l}0.83, \\
0.165\end{array}$ & $\begin{array}{l}0.98, \\
0.016\end{array}$ & $\begin{array}{l}0.93, \\
0.067\end{array}$ & $\begin{array}{l}0.95, \\
0.05\end{array}$ \\
\hline $\mathrm{x}_{3}$ & $\begin{array}{l}0.98 \\
0.021\end{array}$ & $\begin{array}{l}1.0, \\
0.0\end{array}$ & $\begin{array}{l}1.0, \\
0.0\end{array}$ & $\begin{array}{l}0.90, \\
0.095\end{array}$ & $\begin{array}{l}0.79, \\
0.21\end{array}$ & $\begin{array}{l}0.95, \\
0.042\end{array}$ & $\begin{array}{l}0.91, \\
0.09\end{array}$ & $\begin{array}{l}0.98, \\
0.02\end{array}$ & $\begin{array}{l}0.93 \\
0.065\end{array}$ & $\begin{array}{l}0.781, \\
0.22\end{array}$ & $\begin{array}{l}0.83, \\
0.17\end{array}$ & $\begin{array}{l}0.86, \\
0.14\end{array}$ & $\begin{array}{l}1.0, \\
0.0\end{array}$ & $\begin{array}{l}0.95 \\
0.05\end{array}$ & $\begin{array}{l}0.98, \\
0.02\end{array}$ & $\begin{array}{l}0.85, \\
0.15\end{array}$ & $\begin{array}{l}0.88, \\
0.12\end{array}$ & $\begin{array}{l}0.87, \\
0.13\end{array}$ & $\begin{array}{l}0.84, \\
0.16\end{array}$ & $\begin{array}{l}0.93, \\
0.07\end{array}$ \\
\hline$x_{4}$ & $\begin{array}{l}0.98 \\
0.021\end{array}$ & $\begin{array}{l}1.0, \\
0.0\end{array}$ & $\begin{array}{l}0.90, \\
0.095\end{array}$ & $\begin{array}{l}1.0, \\
0.0\end{array}$ & $\begin{array}{l}0.87, \\
0.13\end{array}$ & $\begin{array}{l}0.96, \\
0.041\end{array}$ & $\begin{array}{l}0.90, \\
0.097\end{array}$ & $\begin{array}{l}0.83, \\
0.17\end{array}$ & $\begin{array}{l}0.98 \\
0.015\end{array}$ & $\begin{array}{l}0.78, \\
0.224\end{array}$ & $\begin{array}{l}0.93, \\
0.07\end{array}$ & $\begin{array}{l}1.0, \\
0.02\end{array}$ & $\begin{array}{l}0.95 \\
0.046\end{array}$ & $\begin{array}{l}0.93 \\
0.07\end{array}$ & $\begin{array}{l}0.83, \\
0.166\end{array}$ & $\begin{array}{l}0.88, \\
0.12\end{array}$ & $\begin{array}{l}0.93, \\
0.07\end{array}$ & $\begin{array}{l}0.71, \\
0.29\end{array}$ & $\begin{array}{l}0.86, \\
0.14\end{array}$ & $\begin{array}{l}0.97, \\
0.03\end{array}$ \\
\hline $\mathbf{x}_{5}$ & $\begin{array}{l}0.98 \\
0.021\end{array}$ & $\begin{array}{l}1.0, \\
0.0\end{array}$ & $\begin{array}{l}0.90, \\
0.095\end{array}$ & $\begin{array}{l}0.87, \\
0.13\end{array}$ & $\begin{array}{l}1.0, \\
0.0\end{array}$ & $\begin{array}{l}0.96, \\
0.04\end{array}$ & $\begin{array}{l}0.91, \\
0.09\end{array}$ & $\begin{array}{l}0.88, \\
0.12\end{array}$ & $\begin{array}{l}0.89, \\
0.11\end{array}$ & $\begin{array}{l}0.85, \\
0.15\end{array}$ & $\begin{array}{l}0.90, \\
0.10\end{array}$ & $\begin{array}{l}0.93, \\
0.068\end{array}$ & $\begin{array}{l}0.90, \\
0.095\end{array}$ & $\begin{array}{l}0.95, \\
0.048\end{array}$ & $\begin{array}{l}0.91, \\
0.087\end{array}$ & $\begin{array}{l}0.98, \\
0.02\end{array}$ & $\begin{array}{l}0.93, \\
0.07\end{array}$ & $\begin{array}{l}0.98, \\
0.02\end{array}$ & $\begin{array}{l}0.99, \\
0.014\end{array}$ & $\begin{array}{l}0.60, \\
0.40\end{array}$ \\
\hline$\overline{x_{6}}$ & $\begin{array}{l}0.98, \\
0.021\end{array}$ & $\begin{array}{l}1.0, \\
0.0\end{array}$ & $\begin{array}{l}0.90, \\
0.095\end{array}$ & $\begin{array}{l}0.87, \\
0.13\end{array}$ & $\begin{array}{l}0.96, \\
0.04\end{array}$ & $\begin{array}{l}1.0, \\
0.0\end{array}$ & $\begin{array}{l}0.95, \\
0.045\end{array}$ & $\begin{array}{l}0.94, \\
0.06\end{array}$ & $\begin{array}{l}0.97, \\
0.032\end{array}$ & $\begin{array}{l}0.96, \\
0.04\end{array}$ & $\begin{array}{l}0.97, \\
0.03\end{array}$ & $\begin{array}{l}0.90, \\
0.097\end{array}$ & $\begin{array}{l}0.80, \\
0.20\end{array}$ & $\begin{array}{l}0.87, \\
0.13\end{array}$ & $\begin{array}{l}0.81, \\
0.19\end{array}$ & $\begin{array}{l}0.94, \\
0.06\end{array}$ & $\begin{array}{l}0.94, \\
0.06\end{array}$ & $\begin{array}{l}0.85, \\
0.15\end{array}$ & $\begin{array}{l}0.75, \\
0.25\end{array}$ & $\begin{array}{l}0.83, \\
0.17\end{array}$ \\
\hline $\mathbf{x}_{7}$ & $\begin{array}{l}0.98 \\
0.021\end{array}$ & $\begin{array}{l}1.0, \\
0.0\end{array}$ & $\begin{array}{l}0.90, \\
0.095\end{array}$ & $\begin{array}{l}0.87, \\
0.13\end{array}$ & $\begin{array}{l}0.96, \\
0.04\end{array}$ & $\begin{array}{l}0.95, \\
0.045\end{array}$ & $\begin{array}{l}1.0, \\
0.0\end{array}$ & $\begin{array}{l}0.73, \\
0.266\end{array}$ & $\begin{array}{l}0.88, \\
0.12\end{array}$ & $\begin{array}{l}0.75, \\
0.25\end{array}$ & $\begin{array}{l}0.88, \\
0.12\end{array}$ & $\begin{array}{l}0.68 \\
0.322\end{array}$ & $\begin{array}{l}0.11, \\
0.89\end{array}$ & $\begin{array}{l}0.96, \\
0.04\end{array}$ & $\begin{array}{l}0.83, \\
0.165\end{array}$ & $\begin{array}{l}0.73, \\
0.265\end{array}$ & $\begin{array}{l}0.98, \\
0.018\end{array}$ & $\begin{array}{l}0.63, \\
0.37\end{array}$ & $\begin{array}{l}0.81, \\
0.19\end{array}$ & $\begin{array}{l}0.86, \\
0.137\end{array}$ \\
\hline $\mathbf{x}_{8}$ & $\begin{array}{l}0.98 \\
0.021\end{array}$ & $\begin{array}{l}1.0, \\
0.0\end{array}$ & $\begin{array}{l}0.90, \\
0.095\end{array}$ & $\begin{array}{l}0.87, \\
0.13\end{array}$ & $\begin{array}{l}0.96, \\
0.04\end{array}$ & $\begin{array}{l}0.95, \\
0.045\end{array}$ & $\begin{array}{l}0.73, \\
0.266\end{array}$ & $\begin{array}{l}1.0, \\
0.0\end{array}$ & $\begin{array}{l}0.60, \\
0.40\end{array}$ & $\begin{array}{l}0.53, \\
0.472\end{array}$ & $\begin{array}{l}0.85, \\
0.153\end{array}$ & $\begin{array}{l}0.96, \\
0.041\end{array}$ & $\begin{array}{l}0.38, \\
0.62\end{array}$ & $\begin{array}{l}0.63, \\
0.365\end{array}$ & $\begin{array}{l}0.71, \\
0.294\end{array}$ & $\begin{array}{l}1.0, \\
0.0\end{array}$ & $\begin{array}{l}0.85, \\
0.154\end{array}$ & $\begin{array}{l}0.90, \\
0.098\end{array}$ & $\begin{array}{l}0.93, \\
0.07\end{array}$ & $\begin{array}{l}0.65, \\
0.35\end{array}$ \\
\hline $\mathbf{x}_{9}$ & $\begin{array}{l}0.98 \\
0.021\end{array}$ & $\begin{array}{l}1.0, \\
0.0\end{array}$ & $\begin{array}{l}0.90, \\
0.095\end{array}$ & $\begin{array}{l}0.87, \\
0.13\end{array}$ & $\begin{array}{l}0.96, \\
0.04\end{array}$ & $\begin{array}{l}0.95, \\
0.045\end{array}$ & $\begin{array}{l}0.73, \\
0.266\end{array}$ & $\begin{array}{l}0.60, \\
0.40\end{array}$ & $\begin{array}{l}1.0, \\
0.0\end{array}$ & $\begin{array}{l}0.87, \\
0.13\end{array}$ & $\begin{array}{l}0.76 \\
0.24\end{array}$ & $\begin{array}{l}0.56, \\
0.44\end{array}$ & $\begin{array}{l}0.93 \\
0.07\end{array}$ & $\begin{array}{l}0.93 \\
0.07\end{array}$ & $\begin{array}{l}0.91, \\
0.08\end{array}$ & $\begin{array}{l}0.86, \\
0.14\end{array}$ & $\begin{array}{l}0.96, \\
0.041\end{array}$ & $\begin{array}{l}0.95, \\
0.049\end{array}$ & $\begin{array}{l}0.68, \\
0.317\end{array}$ & $\begin{array}{l}0.93, \\
0.066\end{array}$ \\
\hline $\mathbf{x}_{10}$ & $\begin{array}{l}0.98 \\
0.021\end{array}$ & $\begin{array}{l}1.0, \\
0.0\end{array}$ & $\begin{array}{l}0.90, \\
0.095\end{array}$ & $\begin{array}{l}0.87, \\
0.13\end{array}$ & $\begin{array}{l}0.96, \\
0.04\end{array}$ & $\begin{array}{l}0.95, \\
0.045\end{array}$ & $\begin{array}{l}0.73, \\
0.266\end{array}$ & $\begin{array}{l}0.60, \\
0.40\end{array}$ & $\begin{array}{l}0.87, \\
0.13\end{array}$ & $\begin{array}{l}1.0, \\
1.0\end{array}$ & $\begin{array}{l}0.98, \\
0.022\end{array}$ & $\begin{array}{l}0.71, \\
0.292\end{array}$ & $\begin{array}{l}0.81, \\
0.192\end{array}$ & $\begin{array}{l}0.80, \\
0.20\end{array}$ & $\begin{array}{l}0.85, \\
0.15\end{array}$ & $\begin{array}{l}0.97, \\
0.034\end{array}$ & $\begin{array}{l}0.70, \\
0.295\end{array}$ & $\begin{array}{l}0.73, \\
0.27\end{array}$ & $\begin{array}{l}0.90, \\
0.10\end{array}$ & $\begin{array}{l}0.83, \\
0.17\end{array}$ \\
\hline $\mathbf{x}_{11}$ & $\begin{array}{l}0.98 \\
0.021\end{array}$ & $\begin{array}{l}1.0, \\
0.0\end{array}$ & $\begin{array}{l}0.90, \\
0.095\end{array}$ & $\begin{array}{l}0.87, \\
0.13\end{array}$ & $\begin{array}{l}0.96, \\
0.04\end{array}$ & $\begin{array}{l}0.95, \\
0.045\end{array}$ & $\begin{array}{l}0.73, \\
0.266\end{array}$ & $\begin{array}{l}0.60, \\
0.40\end{array}$ & $\begin{array}{l}0.87, \\
0.13\end{array}$ & $\begin{array}{l}0.98, \\
0.022\end{array}$ & $\begin{array}{l}1.0, \\
0.0\end{array}$ & $\begin{array}{l}0.81 \\
0.194\end{array}$ & $\begin{array}{l}0.83, \\
0.17\end{array}$ & $\begin{array}{l}0.88, \\
0.122\end{array}$ & $\begin{array}{l}0.86, \\
0.139\end{array}$ & $\begin{array}{l}0.85, \\
0.153\end{array}$ & $\begin{array}{l}0.71, \\
0.286\end{array}$ & $\begin{array}{l}0.75, \\
0.25\end{array}$ & $\begin{array}{l}0.93, \\
0.07\end{array}$ & $\begin{array}{l}0.48, \\
0.52\end{array}$ \\
\hline $\mathrm{x}_{12}$ & $\begin{array}{l}0.98, \\
0.021\end{array}$ & $\begin{array}{l}1.0, \\
0.0\end{array}$ & $\begin{array}{l}0.90, \\
0.095\end{array}$ & $\begin{array}{l}0.87, \\
0.13\end{array}$ & $\begin{array}{l}0.96, \\
0.04\end{array}$ & $\begin{array}{l}0.95, \\
0.045\end{array}$ & $\begin{array}{l}0.73, \\
0.266\end{array}$ & $\begin{array}{l}0.60, \\
0.40\end{array}$ & $\begin{array}{l}0.87, \\
0.13\end{array}$ & $\begin{array}{l}0.98, \\
0.022\end{array}$ & $\begin{array}{l}0.81, \\
0.194\end{array}$ & $\begin{array}{l}1.0, \\
0.0\end{array}$ & $\begin{array}{l}0.43, \\
0.57\end{array}$ & $\begin{array}{l}0.68, \\
0.32\end{array}$ & $\begin{array}{l}0.45, \\
0.55\end{array}$ & $\begin{array}{l}0.96, \\
0.04\end{array}$ & $\begin{array}{l}0.90, \\
0.20\end{array}$ & $\begin{array}{l}0.95, \\
0.05\end{array}$ & $\begin{array}{l}0.88, \\
0.12\end{array}$ & $\begin{array}{l}0.68, \\
0.32\end{array}$ \\
\hline $\mathbf{x}_{13}$ & $\begin{array}{l}0.98, \\
0.021\end{array}$ & $\begin{array}{l}1.0, \\
0.0\end{array}$ & $\begin{array}{l}0.90, \\
0.095\end{array}$ & $\begin{array}{l}0.87, \\
0.13\end{array}$ & $\begin{array}{l}0.96, \\
0.04\end{array}$ & $\begin{array}{l}0.95, \\
0.045\end{array}$ & $\begin{array}{l}0.73, \\
0.266\end{array}$ & $\begin{array}{l}0.60, \\
0.40\end{array}$ & $\begin{array}{l}0.87, \\
0.13\end{array}$ & $\begin{array}{l}0.98, \\
0.022\end{array}$ & $\begin{array}{l}0.81, \\
0.194\end{array}$ & $\begin{array}{l}0.43, \\
0.57\end{array}$ & $\begin{array}{l}1.0, \\
0.0\end{array}$ & $\begin{array}{l}0.75, \\
0.25\end{array}$ & $\begin{array}{l}0.98, \\
0.015\end{array}$ & $\begin{array}{l}0.68, \\
0.32\end{array}$ & $\begin{array}{l}0.53, \\
0.47\end{array}$ & $\begin{array}{l}0.48, \\
0.52\end{array}$ & $\begin{array}{l}0.30, \\
0.70\end{array}$ & $\begin{array}{l}0.75, \\
0.25\end{array}$ \\
\hline $\mathbf{x}_{14}$ & 0.98 & 1.0, & 0.90 & 0.87 & 0.96 & 0.95 & 0.73 & 0.60, & 0.87 & 0.98 & 0.81, & 0.43 & 0.75 & 1.0 & 0.78 & 0.63 & 0.78 & 0.73 & 0.55, & 1.0 \\
\hline
\end{tabular}




\begin{tabular}{|c|c|c|c|c|c|c|c|c|c|c|c|c|c|c|c|c|c|c|c|c|}
\hline & 0.021 & 0.0 & 0.095 & 0.13 & 0.04 & 0.045 & 0.266 & 0.40 & 0.13 & 0.022 & 0.194 & 0.57 & 0.25 & 0.0 & 0.22 & 0.366 & 0.22 & 0.27 & 0.45 & \begin{tabular}{ll|}
0.0 \\
\end{tabular} \\
\hline$x_{15}$ & $\begin{array}{l}0.98 \\
0.021\end{array}$ & $\begin{array}{l}1.0, \\
0.0\end{array}$ & $\begin{array}{l}0.90, \\
0.095\end{array}$ & $\begin{array}{l}0.87, \\
0.13\end{array}$ & $\begin{array}{l}0.96, \\
0.04\end{array}$ & $\begin{array}{l}0.95 \\
0.045\end{array}$ & $\begin{array}{l}0.73, \\
0.266\end{array}$ & $\begin{array}{l}0.60, \\
0.40\end{array}$ & $\begin{array}{l}0.87, \\
0.13\end{array}$ & $\begin{array}{l}0.98, \\
0.022\end{array}$ & $\begin{array}{l}0.81, \\
0.194\end{array}$ & $\begin{array}{l}0.43, \\
0.57\end{array}$ & $\begin{array}{l}0.75, \\
0.25\end{array}$ & $\begin{array}{l}0.78, \\
0.22\end{array}$ & $\begin{array}{l}1.0, \\
0.0\end{array}$ & $\begin{array}{l}0.61, \\
0.39\end{array}$ & $\begin{array}{l}0.55, \\
0.45\end{array}$ & $\begin{array}{l}0.51, \\
0.49\end{array}$ & $\begin{array}{l}0.43, \\
0.566\end{array}$ & $\begin{array}{l}0.78, \\
0.22\end{array}$ \\
\hline $\mathbf{X}_{16}$ & $\begin{array}{l}0.98 \\
0.021\end{array}$ & $\begin{array}{l}1.0, \\
0.0\end{array}$ & $\begin{array}{l}0.90, \\
0.095\end{array}$ & $\begin{array}{l}0.87, \\
0.13\end{array}$ & $\begin{array}{l}0.96, \\
0.04\end{array}$ & $\begin{array}{l}0.95, \\
0.045\end{array}$ & $\begin{array}{l}0.73, \\
0.266\end{array}$ & $\begin{array}{l}0.60, \\
0.40\end{array}$ & $\begin{array}{l}0.87, \\
0.13\end{array}$ & $\begin{array}{l}0.98, \\
0.022\end{array}$ & $\begin{array}{l}0.81, \\
0.194\end{array}$ & $\begin{array}{l}0.43, \\
0.57\end{array}$ & $\begin{array}{l}0.75, \\
0.25\end{array}$ & $\begin{array}{l}0.78, \\
0.22\end{array}$ & $\begin{array}{l}0.61, \\
0.39\end{array}$ & $\begin{array}{l}1.0, \\
0.0\end{array}$ & $\begin{array}{l}0.85, \\
0.15\end{array}$ & $\begin{array}{l}0.90, \\
0.10\end{array}$ & $\begin{array}{l}0.93, \\
0.07\end{array}$ & $\begin{array}{l}0.63, \\
0.37\end{array}$ \\
\hline $\mathbf{x}_{17}$ & $\begin{array}{l}0.98 \\
0.021\end{array}$ & $\begin{array}{l}1.0, \\
0.0\end{array}$ & $\begin{array}{l}0.90, \\
0.095\end{array}$ & $\begin{array}{l}0.87, \\
0.13\end{array}$ & $\begin{array}{l}0.96, \\
0.04\end{array}$ & $\begin{array}{l}0.95 \\
0.045\end{array}$ & $\begin{array}{l}0.73, \\
0.266\end{array}$ & $\begin{array}{l}0.60, \\
0.40\end{array}$ & $\begin{array}{l}0.87, \\
0.13\end{array}$ & $\begin{array}{l}0.98, \\
0.022\end{array}$ & $\begin{array}{l}0.81, \\
0.194\end{array}$ & $\begin{array}{l}0.43, \\
0.57\end{array}$ & $\begin{array}{l}0.75, \\
0.25\end{array}$ & $\begin{array}{l}0.78, \\
0.22\end{array}$ & $\begin{array}{l}0.61, \\
0.39\end{array}$ & $\begin{array}{l}0.85, \\
0.15\end{array}$ & $\begin{array}{l}1.0, \\
0.0\end{array}$ & $\begin{array}{l}0.96, \\
0.04\end{array}$ & $\begin{array}{l}0.78, \\
0.22\end{array}$ & $\begin{array}{l}0.78, \\
0.22\end{array}$ \\
\hline $\mathrm{X}_{18}$ & $\begin{array}{l}0.98, \\
0.021\end{array}$ & $\begin{array}{l}1.0, \\
0.0\end{array}$ & $\begin{array}{l}0.90, \\
0.095\end{array}$ & $\begin{array}{l}0.87, \\
0.13\end{array}$ & $\begin{array}{l}0.96, \\
0.04\end{array}$ & $\begin{array}{l}0.95 \\
0.045\end{array}$ & $\begin{array}{l}0.73, \\
0.266\end{array}$ & $\begin{array}{l}0.60, \\
0.40\end{array}$ & $\begin{array}{l}0.87, \\
0.13\end{array}$ & $\begin{array}{l}0.98, \\
0.022\end{array}$ & $\begin{array}{l}0.81, \\
0.194\end{array}$ & $\begin{array}{l}0.43, \\
0.57\end{array}$ & $\begin{array}{l}0.75, \\
0.25\end{array}$ & $\begin{array}{l}0.78, \\
0.22\end{array}$ & $\begin{array}{l}0.61, \\
0.39\end{array}$ & $\begin{array}{l}0.85, \\
0.15\end{array}$ & $\begin{array}{l}0.96, \\
0.04\end{array}$ & $\begin{array}{l}1.0, \\
0.0\end{array}$ & $\begin{array}{l}0.83, \\
0.17\end{array}$ & $\begin{array}{l}0.73, \\
0.27\end{array}$ \\
\hline $\mathbf{x}_{19}$ & $\begin{array}{l}0.98 \\
0.021\end{array}$ & $\begin{array}{l}1.0, \\
0.0\end{array}$ & $\begin{array}{l}0.90, \\
0.095\end{array}$ & $\begin{array}{l}0.87, \\
0.13\end{array}$ & $\begin{array}{l}0.96, \\
0.04\end{array}$ & $\begin{array}{l}0.95 \\
0.045\end{array}$ & $\begin{array}{l}0.73, \\
0.266\end{array}$ & $\begin{array}{l}0.60, \\
0.40\end{array}$ & $\begin{array}{l}0.87, \\
0.13\end{array}$ & $\begin{array}{l}0.98 \\
0.022\end{array}$ & $\begin{array}{l}0.81, \\
0.194\end{array}$ & $\begin{array}{l}0.43, \\
0.57\end{array}$ & $\begin{array}{l}0.75, \\
0.25\end{array}$ & $\begin{array}{l}0.78, \\
0.22\end{array}$ & $\begin{array}{l}0.61, \\
0.39\end{array}$ & $\begin{array}{l}0.85, \\
0.15\end{array}$ & $\begin{array}{l}0.96, \\
0.04\end{array}$ & $\begin{array}{l}0.83, \\
0.17\end{array}$ & $\begin{array}{l}1.0, \\
0.0\end{array}$ & $\begin{array}{l}0.95, \\
0.051\end{array}$ \\
\hline $\mathbf{x}_{20}$ & $\begin{array}{l}0.98 \\
0.021\end{array}$ & $\begin{array}{l}1.0, \\
0.0\end{array}$ & $\begin{array}{l}0.90, \\
0.095\end{array}$ & $\begin{array}{l}0.87, \\
0.13\end{array}$ & $\begin{array}{l}0.96, \\
0.04\end{array}$ & $\begin{array}{l}0.95 \\
0.045\end{array}$ & $\begin{array}{l}0.73, \\
0.266\end{array}$ & $\begin{array}{l}0.60, \\
0.40\end{array}$ & $\begin{array}{l}0.87, \\
0.13\end{array}$ & $\begin{array}{l}0.98 \\
0.022\end{array}$ & $\begin{array}{l}0.81, \\
0.194\end{array}$ & $\begin{array}{l}0.43, \\
0.57\end{array}$ & $\begin{array}{l}0.75, \\
0.25\end{array}$ & $\begin{array}{l}0.78, \\
0.22\end{array}$ & $\begin{array}{l}0.61, \\
0.39\end{array}$ & $\begin{array}{l}0.85, \\
0.15\end{array}$ & $\begin{array}{l}0.96, \\
0.04\end{array}$ & $\begin{array}{l}0.83, \\
0.17\end{array}$ & $\begin{array}{l}0.95 \\
0.051\end{array}$ & $\begin{array}{l}1.0, \\
0.0\end{array}$ \\
\hline
\end{tabular}

By considering the almost similarity of $90 \%$ and dissimilarity of $10 \%$, it is observed from Table4. 1 that $\mathrm{R}\left(\mathrm{x}_{1}, \mathrm{x}_{1}\right)=1.0,0 ; \mathrm{R}\left(\mathrm{x}_{2}, \mathrm{x}_{5}\right)=0.97,0.009 ; \mathrm{R}\left(\mathrm{x}_{3}, \mathrm{x}_{6}\right)=0.99,0.014 ; \mathrm{R}($ $\left.\mathrm{x}_{4}, \mathrm{x}_{7}\right)=0.99,0.0007 ; \mathrm{R}\left(\mathrm{x}_{5}, \mathrm{x}_{8}\right)=0.96,0.036 ; \mathrm{R}\left(\mathrm{x}_{6}, \mathrm{x}_{9}\right)=0.97,0.03 ; \mathrm{R}\left(\mathrm{x}_{7}, \mathrm{x}_{10}\right)=1.0,0.002 ; \mathrm{R}\left(\mathrm{x}_{8}, \mathrm{x}_{11}\right)$ $=0.99,0.007 ; \mathrm{R}\left(\mathrm{x}_{9}, \mathrm{x}_{12}\right)=0.98,0.018 ; \mathrm{R}\left(\mathrm{x}_{10}, \mathrm{x}_{11}\right)=0.99,0.005 ; \mathrm{R}\left(\mathrm{x}_{11}, \mathrm{x}_{12}\right)=0.99,0.008 ; \mathrm{R}\left(\mathrm{x}_{12}, \mathrm{x}_{13}\right)=$ $0.95,0.04 ; \mathrm{R}\left(\mathrm{x}_{13}, \mathrm{x}_{14}\right)=0.98,0.019 ; \mathrm{R}\left(\mathrm{x}_{14}, \mathrm{x}_{15}\right)=1,0.002 ; \mathrm{R}\left(\mathrm{x}_{15}, \mathrm{x}_{16}\right)=0.99,0.005 ; \mathrm{R}\left(\mathrm{x}_{16}, \mathrm{x}_{17}\right)=0.97$, $0.031 ; \mathrm{R}\left(\mathrm{x}_{17}, \mathrm{x}_{18}\right)=0.99,0.014 ; \mathrm{R}\left(\mathrm{x}_{18}, \mathrm{x}_{19}\right)=1,0.003 ; \mathrm{R}\left(\mathrm{x}_{19}, \mathrm{x}_{20}\right)=0.99,0.006 ; \mathrm{R}\left(\mathrm{x}_{1}, \mathrm{x}_{20}\right)=0.95,0.05$. Thus the samples are alpha $(\alpha)$ and beta $(\beta)$ identical. Therefore we get $\mathrm{U} / \mathrm{EC}=\left\{\mathrm{x}_{1}, \mathrm{x}_{2}, \mathrm{x}_{3}, \mathrm{x}_{4}, \mathrm{x}_{5}, \mathrm{x}_{6}, \mathrm{x}_{7}, \mathrm{x}_{8}, \mathrm{x}_{9}, \mathrm{x}_{10}, \mathrm{x}_{11}, \mathrm{x}_{12}, \mathrm{x}_{13}, \mathrm{x}_{14}, \mathrm{x}_{15}, \mathrm{x}_{16}, \mathrm{x}_{17}, \mathrm{x}_{18}, \mathrm{x}_{19}, \mathrm{x}_{20}\right\}$.

From the observations of Algorithm I, the attributes $\{\mathrm{U} / \mathrm{P}, \mathrm{U} / \mathrm{K}, \mathrm{U} / \mathrm{Cu}, \mathrm{U} / \mathrm{Fe}\}$ and $\{\mathrm{U} / \mathrm{N}\}$ classifies the soil samples into two and three groups respectively. Since the equivalence classes $\{\mathrm{U} / \mathrm{Ec}, \mathrm{U} / \mathrm{PH}, \mathrm{U} / \mathrm{Zn}, \mathrm{U} / \mathrm{Mn}\}$ contains only one group, the soil samples are indiscernible according to the attribute set and hence do not require any ordering while extracting knowledge from the information system. The ordered information table of the soil samples (Table 2) is given in Table 5.

Table 5. Intuitionistic Fuzzy Sets and Ordering

\begin{tabular}{|c|c|c|}
\hline & Intuitionistic fuzzy sets & Ordering \\
\hline $\mathrm{U} / \mathrm{Ec}$ & $\left\{\mathrm{i}_{1}, \mathrm{i}_{2}, \mathrm{i}_{3}, \mathrm{i}_{4}, \mathrm{i}_{5}, \mathrm{i}_{6}, \mathrm{i}_{7}, \mathrm{i}_{8}, \mathrm{i}_{9}, \mathrm{i}_{10}, \mathrm{i}_{11}, \mathrm{i}_{12}, \mathrm{i}_{13}, \mathrm{i}_{14}, \mathrm{i}_{15}, \mathrm{i}_{16}, \mathrm{i}_{17}, \mathrm{i}_{18}, \mathrm{i}_{19}, \mathrm{i}_{20}\right\}$ & $<\{0\}$ normal \\
\hline $\mathrm{U} / \mathrm{PH}$ & $\left\{\mathrm{i}_{1}, \mathrm{i}_{2}, \mathrm{i}_{3}, \mathrm{i}_{4}, \mathrm{i}_{5}, \mathrm{i}_{6}, \mathrm{i}_{7}, \mathrm{i}_{8}, \mathrm{i}_{9}, \mathrm{i}_{10}, \mathrm{i}_{11}, \mathrm{i}_{12}, \mathrm{i}_{13}, \mathrm{i}_{14}, \mathrm{i}_{15}, \mathrm{i}_{16}, \mathrm{i}_{17}, \mathrm{i}_{18}, \mathrm{i}_{19}, \mathrm{i}_{20}\right\}$ & $<\{0\}$ normal \\
\hline $\mathrm{U} / \mathrm{N}$ & $\left\{\mathrm{i}_{2}, \mathrm{i}_{8}, \mathrm{i}_{17}\right\},\left\{, \mathrm{i}_{1}, \mathrm{i}_{3}, \mathrm{i}_{4}, \mathrm{i}_{5}, \mathrm{i}_{6}, \mathrm{i}_{7}, \mathrm{i}_{9}, \mathrm{i}_{10}, \mathrm{i}_{11}, \mathrm{i}_{13}, \mathrm{i}_{14}, \mathrm{i}_{15}, \mathrm{i}_{16}, \mathrm{i}_{18}, \mathrm{i}_{20}\right\},\left\{\mathrm{i}_{12}, \mathrm{i}_{19}\right\}$ & $\begin{array}{c}<_{\{1\} \text { low }}<\{2\} \text { medium }< \\
\{3\} \text { high }\end{array}$ \\
\hline $\mathrm{U} / \mathrm{P}$ & $\left\{\mathrm{i}_{3}, \mathrm{i}_{4}, \mathrm{i}_{5}, \mathrm{i}_{6}, \mathrm{i}_{11}, \mathrm{i}_{12}, \mathrm{i}_{14}, \mathrm{i}_{15}, \mathrm{i}_{18}\right\},\left\{\left\{_{\mathrm{i} 1, \mathrm{i} 2, \mathrm{i} 7}, \mathrm{i}_{19}, \mathrm{i}_{10}, \mathrm{i}_{13}, \mathrm{i}_{16}, \mathrm{i}_{17}, \mathrm{i}_{19}, \mathrm{i}_{20}\right\}\right.$ & $<_{\{1\} \text { low }}<_{\{2\} \text { medium }}$ \\
\hline $\mathrm{U} / \mathrm{K}$ & $\left\{\mathrm{i}_{1}, \mathrm{i}_{2}, \mathrm{i}_{3}, \mathrm{i}_{4}, \mathrm{i}_{5}, \mathrm{i}_{6}, \mathrm{i}_{7}, \mathrm{i}_{9}, \mathrm{i}_{10}, \mathrm{i}_{11}, \mathrm{i}_{12}, \mathrm{i}_{13}, \mathrm{i}_{14}, \mathrm{i}_{15}, \mathrm{i}_{16}, \mathrm{i}_{17}\right\},\left\{\mathrm{i}_{\mathrm{i}}, \mathrm{i}_{18}, \mathrm{i}_{19}, \mathrm{i}_{20}\right\}$ & $<_{\{1\} \text { low }}<_{\{2\} \text { medium }}$ \\
\hline $\mathrm{U} / \mathrm{Zn}$ & $\left\{\mathrm{i}_{1}, \mathrm{i}_{2}, \mathrm{i}_{3}, \mathrm{i}_{4}, \mathrm{i}_{5}, \mathrm{i}_{6}, \mathrm{i}_{7}, \mathrm{i}_{8}, \mathrm{i}_{9}, \mathrm{i}_{10}, \mathrm{i}_{11}, \mathrm{i}_{12}, \mathrm{i}_{13}, \mathrm{i}_{14}, \mathrm{i}_{15}, \mathrm{i}_{16}, \mathrm{i}_{17}, \mathrm{i}_{18}, \mathrm{i}_{19}, \mathrm{i}_{20}\right\}$ & $<\{0\}$ optimum \\
\hline $\mathrm{U} / \mathrm{Cu}$ & $\left\{\mathrm{i}_{7}, \mathrm{i}_{20}\right\},\left\{\mathrm{i}_{1}, \mathrm{i}_{2}, \mathrm{i}_{3}, \mathrm{i}_{4}, \mathrm{i}_{5}, \mathrm{i}_{6}, \mathrm{i}_{8}, \mathrm{i}_{9}, \mathrm{i}_{10}, \mathrm{i}_{11}, \mathrm{i}_{12}, \mathrm{i}_{13}, \mathrm{i}_{14}, \mathrm{i}_{15}, \mathrm{i}_{16}, \mathrm{i}_{17}, \mathrm{i}_{18}, \mathrm{i}_{19}\right\}$ & $<_{\{1\} \text { less }}<_{\{2\} \text { optimum }}$ \\
\hline $\mathrm{U} / \mathrm{Fe}$ & $\left\{\mathrm{i}_{3}, \mathrm{i}_{4}, \mathrm{i}_{5}, \mathrm{i}_{6}, \mathrm{i}_{11}, \mathrm{i}_{12}, \mathrm{i}_{13}, \mathrm{i}_{14}, \mathrm{i}_{15}, \mathrm{i}_{16}, \mathrm{i}_{17}, \mathrm{i}_{18}, \mathrm{i}_{19}\right\},\left\{\mathrm{i}_{1}, \mathrm{i}_{2}, \mathrm{i}_{7}, \mathrm{i}_{8}, \mathrm{i}_{9}, \mathrm{i}_{10}, \mathrm{i}_{20}\right\}$ & $<_{\{1\} \text { less }}<\{2\}$ optimum \\
\hline $\mathrm{U} / \mathrm{Mn}$ & $\left\{\mathrm{i}_{1}, \mathrm{i}_{2}, \mathrm{i}_{3}, \mathrm{i}_{4}, \mathrm{i}_{5}, \mathrm{i}_{6}, \mathrm{i}_{7}, \mathrm{i}_{8}, \mathrm{i}_{9}, \mathrm{i}_{10}, \mathrm{i}_{11}, \mathrm{i}_{12}, \mathrm{i}_{13}, \mathrm{i}_{14}, \mathrm{i}_{15}, \mathrm{i}_{16}, \mathrm{i}_{17}, \mathrm{i}_{18}, \mathrm{i}_{19}, \mathrm{i}_{20}\right\}$ & $<\{0\}$ optimum \\
\hline
\end{tabular}

For ordering the soil samples we assign weights to the attribute values using rough set theory. In order to compute the rank $x_{k} ; \mathrm{k}=\{1,2 \ldots .20\}$ we add the weights of the attribute values and rank them according to the total sum obtained from highest to lowest. However, it is identified that the total sum remains same for certain samples. It indicates that these samples cannot be distinguished from one another according to the attribute nutrient values and eliminated from soil fertility ranking. 
Table 6. Ranked Soil Fertility Based on Intuitionistic Proximity Relation

\begin{tabular}{|c|c|c|c|c|c|c|}
\hline Samples & $\mathbf{N}$ & $\mathbf{P}$ & $\mathbf{K}$ & $\mathbf{C u}$ & $\mathbf{F e}$ & Rank \\
\hline $\mathrm{x}_{1}$ & medium(2) & medium(2) & medium(2) & optimum(2) & optimum(2) & 1 \\
\hline $\mathrm{x}_{2}$ & $\operatorname{low}(1)$ & medium(2) & medium(2) & optimum(2) & optimum(2) & 2 \\
\hline $\mathrm{X}_{3}$ & medium(2) & $\operatorname{low}(1)$ & medium(2) & optimum(2) & less(1) & 3 \\
\hline $\mathrm{x}_{4}$ & medium(2) & $\operatorname{low}(1)$ & medium(2) & optimum(2) & less(1) & 3 \\
\hline $\mathrm{X}_{5}$ & medium(2) & $\operatorname{low}(1)$ & medium(2) & optimum(2) & less(1) & 3 \\
\hline $\mathrm{x}_{6}$ & medium(2) & $\operatorname{low}(1)$ & medium(2) & optimum(2) & $\operatorname{less}(1)$ & 3 \\
\hline $\mathrm{x}_{7}$ & medium(2) & medium(2) & medium(2) & less $(1)$ & optimum(2) & 2 \\
\hline $\mathrm{X}_{8}$ & $\operatorname{low}(1)$ & medium(2) & $\operatorname{low}(1)$ & optimum(2) & optimum(2) & 3 \\
\hline $\mathrm{X}_{9}$ & medium(2) & medium(2) & medium(2) & optimum(2) & optimum(2) & 1 \\
\hline $\mathrm{X}_{10}$ & medium(2) & medium(2) & medium(2) & optimum(2) & optimum(2) & 1 \\
\hline $\mathrm{x}_{11}$ & medium(2) & $\operatorname{low}(1)$ & medium(2) & optimum(2) & less(1) & 3 \\
\hline $\mathrm{X}_{12}$ & high (3) & $\operatorname{low}(1)$ & medium(2) & optimum(2) & less(1) & 2 \\
\hline $\mathrm{X}_{13}$ & medium(2) & medium(2) & medium(2) & optimum(2) & less(1) & 2 \\
\hline $\mathrm{X}_{14}$ & medium(2) & $\operatorname{low}(1)$ & medium(2) & optimum(2) & less(1) & 3 \\
\hline $\mathrm{X}_{15}$ & medium(2) & $\operatorname{low}(1)$ & medium(2) & optimum(2) & less(1) & 3 \\
\hline $\mathrm{x}_{16}$ & medium(2) & medium(2) & medium(2) & optimum(2) & less(1) & 2 \\
\hline $\mathrm{X}_{17}$ & low (1) & medium(2) & medium(2) & optimum(2) & less(1) & 3 \\
\hline $\mathrm{X}_{18}$ & medium(2) & $\operatorname{low}(1)$ & $\operatorname{low}(1)$ & optimum(2) & less(1) & 4 \\
\hline $\mathrm{X}_{19}$ & high (3) & medium(2) & $\operatorname{low}(1)$ & optimum(2) & less(1) & 2 \\
\hline $\mathrm{X}_{20}$ & medium(2) & medium(2) & $\operatorname{low}(1)$ & less(1) & optimum(2) & 3 \\
\hline
\end{tabular}

\subsection{Fuzzy Bayesian Based Seasonal Paddy Crop Selection}

In addition with soil fertility, paddy cropping is dependent over a wide range of agroecosystems like varying locations, climate, soil organic matter and ground water level. By scheming requirement parameters such as duration of the crop, grain type and expected average yield we can optimize the requirement of irrigation water, added fertilizer and increase yields for precise seasonal paddy variety. The Fuzzy Bayesian approach allows modeling of seasonal paddy variety selection related uncertainties by providing a symbolic framework for knowledge comprehensibility. The crop selection parameters cannot be directly represented as precise numbers or precisely classified. The Bayesian interprets these parameters as fuzzy data in probability as an extension of propositional logic that enables reasoning with hypotheses, i.e., the propositions whose truth or falsity is uncertain.

The Bayesian decision $[11,12]$ with fuzzy events is represented as a quadruple $<I, S, P$, $U>$ where

$I=\left\{I_{1}, I_{2} \ldots I_{n}\right\}$ be the set of input variables defined over a probabilistic space of $D_{i}$ defined as $I: \mathrm{D}_{i} \rightarrow[0,1]$

$S=\left\langle s_{1}, s_{2}, s_{3} \ldots s_{i}\right\rangle$ be the set of states defined over a probabilistic space of $D_{j}$ defined as

$$
S: \mathrm{D}_{j} \rightarrow[0,1]
$$

$P=\left\{p\left(s_{1}\right), p\left(s_{2}\right) \ldots . p\left(s_{n}\right)\right\}$

$$
\sum_{i=1}^{n} p\left(\mathrm{~s}_{i}\right)=1
$$

$\mathrm{U}=$ utility function on $S \times I$

By Bayes rule, If posterior probability of fuzzy event $\mathrm{P}\left(\mathrm{s}_{\mathrm{i}} / \mathrm{I}_{\mathrm{k}}\right)$ with known piece of information $I_{k}$ is true then, probability that the state of decision is $s_{i}$ is given by eq(1)

$$
P\left(s_{i} / I_{k}\right)=\frac{\mathrm{p}\left(\mathrm{I}_{\mathrm{k}} / \mathrm{s}_{\mathrm{i}}\right) p\left(\mathrm{~s}_{\mathrm{i}}\right)}{\mathrm{p}\left(\mathrm{I}_{\mathrm{k}}\right)}
$$

Where the denominator term is the marginal probability of data $I_{k}$, and is determined by using the total probability theorem. 


$$
p\left(\mathrm{I}_{k}\right)=\sum_{i=1}^{n} p\left(\mathrm{I}_{k} / \mathrm{S}_{i}\right) p\left(s_{i}\right)
$$

The expected utility for the $\mathrm{j}_{\mathrm{th}}$ alternative for the given data $\mathrm{I}_{\mathrm{k}}$ is is given by eq (3)

$$
E\left(u_{j} / I_{k}\right)=\sum_{i=1}^{n} u_{i j} p\left(s_{i} / I_{k}\right)
$$

The maximum expected utility for the given observed data and new data $I_{k}$ is given by eq (4) and (5) respectively

$$
\begin{aligned}
& E\left(u^{*} / I_{k}\right)=E\left(u_{j} / I_{k}\right) p\left(\mathrm{I}_{k}\right) \\
& E\left(u^{*}\right)=\sum_{i=1}^{n} E\left(u_{j} / I_{k}\right) \mathrm{p}\left(\mathrm{I}_{k}\right)
\end{aligned}
$$

The value of information $\mathrm{V}(\mathrm{x})$ in new data can be assessed by difference between maximum expected utility in observed data and the maximum expected utility in new data

$$
V(x)=\left|E\left(u^{*}\right)-E\left(u^{*} / I_{k}\right)\right|
$$

\author{
Algorithm II : Learn from rules for Seasonal crop selection \\ Input : $\mathrm{D}$, a dataset of soil samples \\ $\mathrm{S}_{\mathrm{ij}}$, seasonal paddy variety \\ I, Input Variables: Duration, Average yield, Grain type \\ U, Utility matrix of costs \\ Output: Decision rule set for selective objective: $S_{i j}$ \\ 1. Define fuzzy states for duration, average yield and grain type \\ 2. Define orthogonal fuzzy information system for $S_{i j}$ \\ 3 . For the training data, identify utility matrix and prior probability \\ 4. For the testing data find posterior, conditional probability \\ 5 . Determine the value of information to choose the approximate alternative $S_{i j}$.
}

4.3.1 Illustrative Example: For realizing optimum productivity of paddy in related production environment (Vellore district, Tamilnadu, India) the choice of an appropriate variety is extremely essential. In our study, the criteria considered for paddy variety selection are duration, average yield and grain type. The fuzzy Bayesian decisiontheoretic analysis in Algorithm II adapts a systematic method for determining the precise decision by using more familiar notions of costs (utility matrix) and risks.

Table 7. Salient Paddy Selection Parameters

\begin{tabular}{l|l|l|l|l}
\hline S.No & Duration(days) & $\begin{array}{l}\text { Average Yield } \\
(\mathbf{k g} / \mathbf{h a})\end{array}$ & Grain Type & Variety \\
\hline $\mathrm{x}_{1}$ & 110 & 4000 & medium & ADT36 \\
\hline $\mathrm{X}_{2}$ & 105 & 6200 & short bold & ADT 37 \\
\hline $\mathrm{X}_{3}$ & 115 & 5600 & short bold & ASD 16 \\
\hline $\mathrm{x}_{4}$ & $105-110$ & 5900 & medium slender & ASD 18 \\
\hline $\mathrm{X}_{5}$ & 110 & 4900 & medium slender & ADT 43 \\
\hline $\mathrm{X}_{6}$ & $95-100$ & 4500 & medium slender & MDU 5 \\
\hline $\mathrm{X}_{7}$ & 130 & 6000 & long slender & IR 50 \\
\hline $\mathrm{X}_{8}$ & $110-115$ & 5832 & medium slender & CO47 \\
\hline $\mathrm{X}_{9}$ & 110 & 5400 & medium slender & ADT 45 \\
\hline $\mathrm{x}_{10}$ & 118 & 6200 & medium slender & ADT 47 \\
\hline & & & &
\end{tabular}




\begin{tabular}{l|l|l|l|l}
\hline $\mathrm{x}_{11}$ & $94-99$ & 4800 & long slender & ADT 48 \\
\hline $\mathrm{x}_{12}$ & $110-115$ & 7500 & medium slender & CORH3 \\
\hline
\end{tabular}

4.3.2 Define the Fuzzy States of Nature: In fuzzy set, the input variable is linguistically described or approximately measured based on complexity of situation. Let $\mathrm{I}=\{$ Duration, Average Yield, Grain type be the input variables and the membership functions are derived for each fuzzy set based on the expertise knowledge of crop selection parameters.

\begin{tabular}{clll}
\multicolumn{1}{c}{ Input variables } & \multicolumn{1}{c}{ Fuzzy states } \\
Duration $\left(\mathrm{I}_{1}\right)$ & short & medium & long \\
& $1-105$ days & $106-135$ days & $136-150$ days \\
Average Yield $\left(\mathrm{I}_{2}\right)$ & low & medium & high \\
& $2000-3000 \mathrm{~kg} / \mathrm{ha}$ & $3001-$ & $4000-6200 \mathrm{~kg} / \mathrm{ha}$ \\
Grain Type $\left(\mathrm{I}_{3}\right)$ & Short & $3999 \mathrm{~kg} / \mathrm{ha}$ & \\
& & medium & Long
\end{tabular}

4.3.3 Define Fuzzy Alternatives: Defining the decision alternative helps us to decide the plantation of suitable paddy variety adaptable to average yield, duration of the crop and soil fertility. Let $\mathrm{S}_{\mathrm{ij}}=$ \{Sornavari, Samba, Navarai $\}$ and $\mathrm{S}_{\mathrm{ij}}$ is the representation of selective $j_{\text {th }}$ paddy variety of $i_{\text {th }}$ season subjective to environmental constrains.

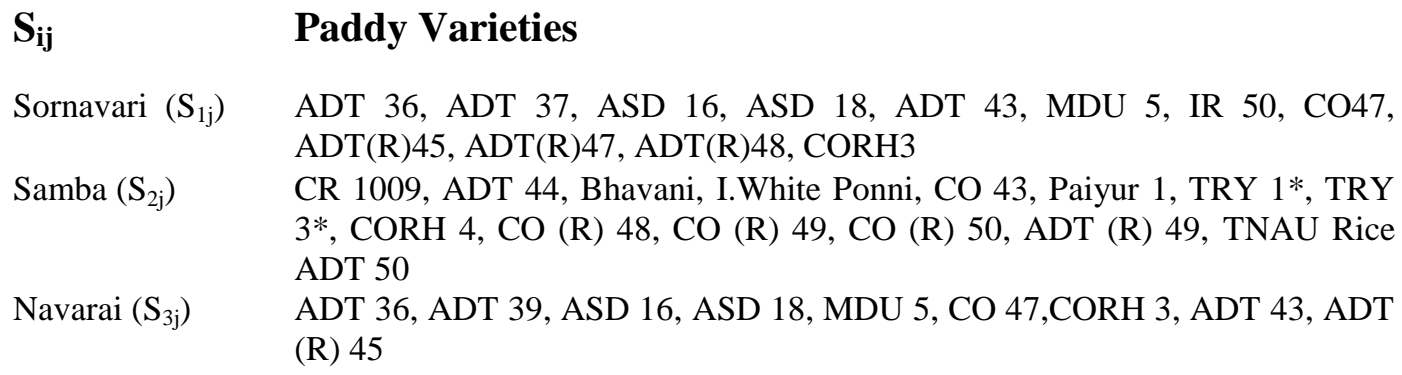

4.3.4 Define New Data Samples: To test the effectiveness of the proposed method, our study considers a target dataset of 70 elements. The data were checked for consistency and completeness. The unreliable and unrelated data were removed to avoid complexity. We randomly divided the 50 datasets into the training data set of 40 records (90\%) and testing data set of 10 records $(10 \%)$.

$\begin{array}{llll}\text { Duration } & \begin{array}{l}\text { Average } \\ \text { Yield }\end{array} & \begin{array}{l}\text { Grain } \\ \text { Type }\end{array} & \text { Variety } \\ 70 & 4000 & \text { medium } & ?\end{array}$

4.3.5Define orthogonal Fuzzy Information System and the Prior Probabilities: Considering the testing data as inherently fuzzy, an orthogonal fuzzy information system $\mathrm{O}\{\mathrm{P}, \mathrm{M}, \mathrm{G}\}=\{$ Poor,Moderate,Good $\}$ with membership values are defined. It means that the sum of the membership values for each fuzzy event I, for every data point in the information universe, $I_{k}$, equals unity.

$\begin{array}{lccc} & \text { Duration } & \text { Average Yield } & \text { Grain Type } \\ \mu_{\mathrm{P}}\left(\mathrm{I}_{\mathrm{k}}\right) & 0.11 & 0.2 & 0.2\end{array}$




$\begin{array}{llll}\mu_{\mathrm{m}}\left(\mathrm{I}_{\mathrm{k}}\right) & 0.8 & 0.4 & 0.8 \\ \mu_{\mathrm{g}}\left(\mathrm{I}_{\mathrm{k}}\right) & 0.09 & 0.6 & 0.2\end{array}$

The prior probability of state $\mathrm{S}_{\mathrm{ij}}$ is computed based on prior expertise knowledge for the training data before adding the testing data. According to expertise knowledge Sonavarai $\left(S_{1 j}\right)$ crop cultivation are more preferable than Samba $\left(S_{2 j}\right)$ and Navarai $\left(S_{3 j}\right)$ crops.

$\begin{array}{ll}\mathbf{S}_{\mathrm{ij}} & \text { Probability } \\ \text { Sornavari } & \mathrm{p}\left(\mathrm{s}_{1 \mathrm{j}}\right)=0.5 \\ \text { Samba } & \mathrm{p}\left(\mathrm{s}_{2 \mathrm{j}}\right)=0.3 \\ \text { Navarai } & \mathrm{p}\left(\mathrm{s}_{3 \mathrm{j}}\right)=0.2\end{array}$

4.3.6 Identify the Utility Values: The utility matrix $U_{i j}$ assigns the utility of choosing alternative $S_{\mathrm{ij}}$ when the inputs of the system is I . The utility function of $I X S$ given in the form of $m \times n$ matrix. Each element in $U_{i j}$ is the cost function following from inputs $I_{i}$ with result $S_{\mathrm{ij}}$

Table 8. Utility Matrix of Costs

\begin{tabular}{|c|c|c|c|c|c|c|c|c|c|c|c|c|}
\hline $\mathbf{U}_{\mathrm{ij}}$ & $\begin{array}{l}\text { ADT } \\
36\end{array}$ & $\begin{array}{l}\text { ADT } \\
37\end{array}$ & $\begin{array}{l}\text { ASD } \\
16\end{array}$ & $\begin{array}{l}\text { ASD } \\
18\end{array}$ & $\begin{array}{l}\text { ADT } \\
\mathbf{4 3}\end{array}$ & $\begin{array}{l}\text { MDU } \\
5\end{array}$ & $\begin{array}{l}\text { IR } \\
50\end{array}$ & CO47 & $\begin{array}{l}\text { ADT } \\
45\end{array}$ & $\begin{array}{l}\text { ADT } \\
47\end{array}$ & $\begin{array}{l}\text { ADT } \\
48\end{array}$ & CORH3 \\
\hline \multicolumn{13}{|c|}{ Duration } \\
\hline short & 5 & 5 & 3 & 1 & 4 & 2 & 5 & 3 & 2 & 2 & 5 & 0 \\
\hline medium & 2 & 2 & 2 & 0 & 2 & 0 & 2 & 1 & 1 & 1 & 1 & 0 \\
\hline long & 0 & 0 & 0 & 0 & 0 & 0 & 0 & 0 & 0 & 0 & 0 & 1 \\
\hline Total & 7 & 7 & 5 & 1 & 6 & 2 & 7 & 4 & 3 & 3 & 6 & 1 \\
\hline \multicolumn{13}{|c|}{ Average Yield } \\
\hline low & 0 & 0 & 0 & 0 & 0 & 0 & 0 & 0 & 0 & 0 & 0 & 0 \\
\hline medium & 4 & 5 & 3 & 3 & 5 & 4 & 4 & 3 & 3 & 3 & 2 & 3 \\
\hline high & 5 & 4 & 0 & 1 & 4 & 1 & 5 & 3 & 1 & 5 & 4 & 0 \\
\hline Total & 9 & 9 & 3 & 4 & 9 & 5 & 9 & 6 & 4 & 8 & 6 & 3 \\
\hline \multicolumn{13}{|c|}{ Grain Type } \\
\hline short & 0 & 5 & 5 & 0 & 0 & 0 & 0 & 0 & 0 & 0 & 0 & 0 \\
\hline medium & 5 & 0 & 0 & 5 & 5 & 5 & 0 & 5 & 5 & 5 & 0 & 5 \\
\hline long & 0 & 0 & 0 & 0 & 0 & 0 & 5 & 0 & 0 & 0 & 5 & 0 \\
\hline Total & 5 & 5 & 5 & 5 & 5 & 5 & 5 & 5 & 5 & 5 & 5 & 5 \\
\hline
\end{tabular}

\subsubsection{Define the Conditional Probabilities of Testing Data:}

Table 9 represents the conditional probability $P\left(I_{k} / s_{i j}\right)$ for uncertain information ie.,te sting data $<$ duration $=\operatorname{short}(70)$,yield $=$ medium $(4000)$ and grain $=$ medium $>$ calculated over the training data which is then used as weights on prior probabilities $\mathrm{p}\left(\mathrm{S}_{\mathrm{ij}}\right)$ to find the updated posterior probability $P\left(s_{i j} / I_{k}\right)$

Table 9. Conditional Probability of Testing Data

\begin{tabular}{c|c|c|c|c|c|c|c|c|c|c|c|c}
\hline$P\left(I_{k} / s_{i j}\right)$ & ADT & ADT & ASD & ASD & ADT & MDU & IR & & ADT & ADT & ADT \\
$\mathbf{3 6}$ & $\mathbf{3 7}$ & $\mathbf{1 6}$ & $\mathbf{1 8}$ & $\mathbf{4 3}$ & $\mathbf{5}$ & $\mathbf{5 0}$ & $\mathbf{C O 4 7}$ & $\begin{array}{c}\mathbf{4 5} \\
\mathbf{4 7}\end{array}$ & $\mathbf{4 8}$ & $\mathbf{\text { CORH3 }}$ \\
\hline $\mathrm{P}\left(\right.$ duration=short $\left./ \mathrm{s}_{1 \mathrm{j}}\right)$ & 0.0 & 0.5 & 0.0 & 0.10 & 0.0 & 0.40 & 0.0 & 0.0 & 0.0 & 0.0 & 0.0 & 0.0 \\
\hline
\end{tabular}




\begin{tabular}{c|c|c|c|c|c|c|c|c|c|c|c|c}
\hline $\mathrm{P}\left(\right.$ yield=medium/ $\left.\mathrm{s}_{2 \mathrm{j}}\right)$ & 0.30 & 0.0 & 0.06 & 0.0 & 0.3 & 0.0 & 0.0 & 0.03 & 0.06 & 0.0 & 0.3 & 0.06 \\
\hline $\mathrm{P}\left(\right.$ grain=medium/ $\left.\mathrm{s}_{3 \mathrm{j}}\right)$ & 0.23 & 0.0 & 0.0 & 0.1 & 0.1 & 0.12 & 0.0 & 0.01 & 0.01 & 0.13 & 0.0 & 0.18 \\
\hline
\end{tabular}

4.3.8 Define the Posterior Probability of Testing Data: Table 10 summarizes calculations for the posterior probabilities $P\left(s_{i j} / I_{k}\right)$, the marginal probabilities $p\left(I_{k}\right)$, the expected conditional utilities $E\left(\mathrm{U} / I_{k}\right)$ and value of uncertainty $v\left(I_{k}\right)$ of the testing data $<$ duration $=\operatorname{short}(70)$, yield $=$ medium $(4000)$ and grain=medium $>$. Typical calculations for values in the table are given below.

Table 10. Posterior Probability of Testing Data

\begin{tabular}{l|c|c|c|c|c|c|c|c|c|c|c|c}
\hline & ADT 36 & ADT 37 & ASD 16 & $\begin{array}{c}\text { ASD } \\
\mathbf{1 8}\end{array}$ & $\begin{array}{c}\text { ADT } \\
\mathbf{4 3}\end{array}$ & $\begin{array}{c}\text { MDU } \\
\mathbf{5}\end{array}$ & $\begin{array}{c}\text { IR } \\
\mathbf{5 0}\end{array}$ & CO47 & ADT 45 & ADT 47 & $\begin{array}{c}\text { ADT } \\
\mathbf{4 8}\end{array}$ & CORH3 \\
\hline $\begin{array}{l}\mathrm{P}\left(\mathrm{s}_{1 \mathrm{j}} /\right. \\
\text { duration=short })\end{array}$ & 0 & 0.3 & 0 & 0.2 & 0 & 0.3 & 0 & 0 & 0 & 0 & 0 & 0 \\
\hline $\begin{array}{l}\mathrm{P}\left(\mathrm{s}_{2 \mathrm{j}} /\right. \\
\text { yield=medium })\end{array}$ & 1 & 0 & 0.2 & 0 & 1 & 0 & 0 & 0.13 & 0.2 & 0 & 1 & 0.2 \\
\hline $\begin{array}{l}\mathrm{P}\left(\mathrm{s}_{3 \mathrm{j}}\right. \\
\text { /grain=medium })\end{array}$ & 1 & 0 & 0 & 0.5 & 0.5 & 0.06 & 0 & 0.05 & 0.05 & 0.65 & 0 & 0.9 \\
\hline $\mathrm{P}\left(\mathrm{I}_{\mathrm{k}}\right)$ & 1 & 0.15 & 0.10 & 0.35 & 0.75 & 0.18 & 0 & 0.09 & 0.125 & 0.325 & 0.5 & 0.55 \\
\hline $\mathrm{E}\left(\mathrm{U} / \mathrm{I}_{\mathrm{k}}\right)$ & 9 & 1.5 & 0.06 & 0.35 & 0.45 & 0.06 & 0 & 0.38 & 0.75 & 3.25 & 2 & 0.51 \\
\hline $\mathrm{E}\left(\mathrm{U}^{*} / \mathrm{I}_{\mathrm{k}}\right)$ & 9 & 0.225 & 0.006 & 0.12 & 0.34 & 0.01 & 0 & 0.03 & 0.09 & 1.05 & 1 & 0.28 \\
\hline $\mathrm{v}\left(\mathrm{I}_{\mathrm{k}}\right)$ & 78.16 & 0 & 0 & 0 & 0 & 0 & 0 & 0 & 0 & 0 & 0 & 0 \\
\hline
\end{tabular}

The marginal probability $p\left(I_{k}\right)$ and posterior probabilities $P\left(s_{i j} / I_{k}\right)$ of the testing data are calculated based on the conditional probabilities $P\left(I_{k} / s_{i j}\right)$ in Table 9 and the prior probabilities $\mathrm{P}\left(\mathrm{S}_{\mathrm{ij}}\right)$.

By equation 1 ,

$P\left(s_{A D T 3}\right.$ d duration $=$ short $)=(0 * 0.5) /(0.5)=0$

$P\left(s_{A D T} 45\right.$ yield $=$ medium $)=(0.06 * 0.3) / 0.07=0.2$

By equation 2 ,

$P\left(I_{k}\right)=0+1(0.5)+1(0.5)=1 ; \quad$ for $A D T 36$

$P\left(I_{k}\right)=0+0.2(0.5)+0.9(0.5)=0.55$; for $C O R H 3$

The expected conditional probability $E\left(U / I_{k}\right)$ is actually the sum of pair wise products of the values in the utility matrix (Table 8 ) and posterior probability values and is calculated using the equation 3 .

$E(U / M D U 5)=0.3(2)+0(0)+0.06(0)=0.06$

$E(U / D T 48)=0(5)+1(2)+0(0)=2$

The overall expected utility for the testing data $I_{k}$ is derived using equation 5 which is sum of pair wise values in row 5,6 of Table 10

$E\left(U^{*}\right)=9(9)+1.5(0.225)+0.06(0.006)+0.35(0.12)+0.45(0.34)+0.06(0.01)+0+0.38(0.0$ $3)+0.75(0.09)+3.25(1.05)+2(1)+0.51(0.28)=87.16$

Finally to decide appropriate alternative to choose in Table 10 , we find $S_{i j}$ with Max $\left.E\left(U^{*} / I_{k}\right)\right) \wedge \operatorname{Max}\left(\sum_{i=1}^{k} U_{i j}\right)$ favoring every element in $\mathrm{I}_{\mathrm{k}}$. For the 
testing data $<$ duration=short, yield=medium and grain=medium $>$ the favorable crop variety is ADT 36 ie., . $\operatorname{Max}\left(E\left(U^{*} / I_{k}\right)\right)=9$ and $\operatorname{Max}\left(\sum_{i=1}^{k} U_{i j}\right)=9+5+5=14$

4.3.9 Determine the Value of Uncertainty in Testing Data: The value of uncertainty in testing data for ADT $36 \quad \varepsilon \quad S_{i j}$ is obtained by equation 6. For $V(<$ duration $=\operatorname{short}(70)$,yield $=$ medium $(4000)$ and grain $=$ medium $>)=87.16-9=78.16$ which is greater than all other value of information $v\left(I_{k}\right)$ so still ADT 36 is the suitable choice of crop variety.

\section{Performance Analysis}

The proposed algorithm works in two phases. The first phase involves the generation of soil fertility and the second phase is the application of combined fuzzy and Bayesian on the objective function to select appropriate paddy variety for cultivation. For fuzzy Bayesian we randomly divided the target data set into two groups called training dataset $(90 \%)$ and testing dataset (10\%).After performing data cleaning and pre processing, the training dataset builds the knowledge mining model and the testing dataset checks the accuracy of variety selection by the hybrid intelligent system. In order to validate our approach we have compared it with some conventional decision making algorithms and the results are shown in Table 11.

Accuracy: It is the degree of correctness to calculate the performance of the system. The higher the accuracy the better the performance of the system.

Accuracy $=\mathrm{TP}+\mathrm{TN} / \mathrm{TP}+\mathrm{TN}+\mathrm{FP}+\mathrm{FN}$

Sensitivity: It is a measure of how a system properly identifies condition and concludes solution. (ie) identifying specific paddy variety under specific selection parameters.

Sensitivity $=\mathrm{TP} / \mathrm{TP}+\mathrm{FN}$

Specificity: It is a measure of how a system properly identifies the wrong solutions. (ie) probability that the system indicates negative selection of paddy varieties.

Specificity $=\mathrm{TN} / \mathrm{TN}+\mathrm{FP}$

Table 11. Performance Evaluation of Target Data

\begin{tabular}{l|l|l|l|l|l|l|l|l}
\hline \multirow{2}{*}{ Method } & \multicolumn{9}{c}{ Training Data } & \multicolumn{3}{c}{ Testing Data } \\
\cline { 2 - 9 } & \multicolumn{9}{|c|}{$\begin{array}{l}\text { Average } \\
\text { Accuracy }\end{array}$} & $\begin{array}{l}\text { Average } \\
\text { Specificity }\end{array}$ & $\begin{array}{l}\text { Average } \\
\text { Sensitivity }\end{array}$ & Samples & $\begin{array}{l}\text { Average } \\
\text { Accuracy }\end{array}$ & $\begin{array}{l}\text { Average } \\
\text { Specificity }\end{array}$ & $\begin{array}{l}\text { Average } \\
\text { Sensitivity }\end{array}$ \\
\hline $\begin{array}{l}\text { Seural } \\
\text { network }\end{array}$ & 70 & $80.7 \%$ & $67 \%$ & $60 \%$ & 10 & $84.2 \%$ & $64 \%$ & $61 \%$ \\
\hline $\begin{array}{l}\text { Fuzzy } \\
\text { bayesian }\end{array}$ & 70 & $90.2 \%$ & $85 \%$ & $70 \%$ & 10 & $93.8 \%$ & $86 \%$ & $73 \%$ \\
\hline $\begin{array}{l}\text { Navie } \\
\text { bayesian }\end{array}$ & 70 & $79.5 \%$ & $77 \%$ & $57 \%$ & 10 & $66.7 \%$ & $77 \%$ & $57 \%$ \\
\hline
\end{tabular}

\section{Conclusion}

The universe of discourse represents real time decision problems involving continuous or mixture of discrete and continuous variables. These variables are modeled using linguistic hedges of fuzzy numbers .A variety of statistical analysis methods fed into decision methodology provides good approximate solutions for moderate sized problems. Intuitionistic fuzzy rough and fuzzy bayesian sapproach of uncertainty management solves conceptual and inferential issues of high structural and high dimensional domains. 
Our work provides a framework that allows us to systematically explore options, develop and examine beliefs. This methodology not only helps to make a considered choice but develop arguments explaining why the policy was chosen in a logical and consistent manner. The simulation was tested real time against the soil test samples of Vellore district, Tamilnadu, India and the results proved consistent precision and accuracy than other conventional decision making techniques.

\section{References}

[1] Atanassov K. T., "Intuitionistic fuzzy sets," Fuzzy Sets and Systems, vol. 20, (1986), pp. 87-96.

[2] Pawlak Z. and Skowron A., "Rudiments of rough sets," Information Science, An International Journal, vol. 177 no. 1, (2007), pp. 3-27.

[3] De C., Radzikowska A. M. and Kerre E. E., "A fuzzy-rough approach to the representation of linguistic hedges," Technologies for Constructing Intelligent Systems 1 Studies in Fuzziness and Soft Computing, vol. 89, (2002), pp. 33-42

[4] Garg H., "An approach for analyzing fuzzy system reliability using particle swarm optimization and intuitionistic fuzzy set theory," Journal of Multiple-Valued Logic and Soft Computing, vol. 3-4, (2013), pp. 335-354

[5] Zeng A., Pan D., Zheng Q. L., "Knowledge Acquisition Based on Rough Set Theory and Principal Component Analysis," IEEE Intelligent Systems, (2006), pp. 78 - 85.

[6] Hsiao C. C., Yi W. K., "A predictor from numerical data based on fuzzy sets and rough sets," 3rd International Workshop on Advanced Computational Intelligence, IWACI, Suzhou, China, August 2527, (2010), pp. 139-144.

[7] Xu Z. S., Yager R. R., "Some geometric aggregation operators based on intuitionistic fuzzy ," sets.International Journal of General Systems, vol. 35, (2006), pp. 417-433.

[8] Deschrijver G., Kerre E., “Aggregation operators in interval-valued fuzzy and Atanassov's intuitionistic fuzzy set theory," Studies in Fuzziness and Soft Computing, vol. 220, (2008), pp. 183-203.

[9] Kucukvar M., "Ranking the sustainability performance of pavements: An intuitionistic fuzzy decision making method," Automation in Construction, vol. 40, (2014), pp. 33-43.

[10] Cesar A. P., Carlos A. F., Silvia M. N., Rodolfo C.C. F. and Marco A. D. O., "Fuzzy-Bayesian network for refrigeration compressor performance prediction and test time reduction," Expert Systems with Applications, vol. 39 no. 4, (2012), pp. 4268-4273.

[11] Liessman S. and John S., "Principal component analysis preprocessing with bayesian networks for battery capacity estimation," I2MTC, IEEE, (2013), pp. 98-101.

[12] Cagman N., Enginoglu S. and Citak F., "Fuzzy Soft Set Theory and Its Applications," Iran Journal of Fuzzy systems, vol. 8 no. 3, (2011), pp. 137-147.

[13] Jiang Y. and Tang Y. C., "An adjustable approach to intuitionistic fuzzy soft sets based decision making," Applied Mathematical Modeling, vol. 35, (2011), pp. 824-836.

[14] "http://agritech.tnau.ac.in/agriculture/agri_resourcemgt_soil.html."

[15] Kucukvar M., Gumus S. E. G. and Tatari O., "Ranking the sustainability performance of pavements: An intuitionistic fuzzy decision making method," Automation in Construction, vol. 40, (2014), pp. 33-43.

[16] Chu C. H., Hung K. C. and Julian P., "A complete pattern recognition approach under Atanassov's intuitionistic fuzzy sets," Knowledge-Based Systems, vol. 66, (2014), pp. 36-45.

[17] Shu P. W. and Deng F. L., "Possibility mean and variance based method for multi-attribute decision making with triangular intuitionistic fuzzy numbers," Journal of Intelligent and Fuzzy Systems, vol. 24 no. 4, (2013), pp. 743-754.

[18] Klaus S., "Revolution in the paddy field," Appropriate Technology, vol. 39 no. 4, (2012), pp. 38-41.

[19] Polat S., Aksoy A. and Unlu K., "A fuzzy rule based remedial priority ranking system for contaminated sites," Groundwater, May 19, (2014).

[20] Mueller L., Shepherd G., Schindler U., Ball B. C., Munkholm L. J., Hennings V., Smolentseva E., Rukhovic O., Lukin S. and Hu C., "Evaluation of soil structure in the framework of an overall soil quality rating," Soil and Tillage Research, vol. 127, (2013), pp. 74-84.

[21] Pilbeam C. J., Mathema B., Gregory P. J. and Shakya P. B., "Soil fertility management in the mid-hills of Nepal: Practices and perceptions," Agriculture and Human Values, vol. 22 no. 2, (2005), pp. 243-258.

[22] Allan L. J., "Building a web-based information system for variety selection in field crops-objectives and results," Computers and Electronics in Agriculture, vol. 32 no. 3, (2001), pp. 195-211.

[23] Lee W. S., Alchanatis V., Yang C., Hirafuji M., Moshou D. and Li C., "Sensing technologies for precision specialty crop production," Computers and Electronics in Agriculture, vol. 74 no. 1, (2010), pp. 2-33.

[24] Marta C. P. and Carlos J. A. L., "Model for decision-making in agricultural production planning," Computers and Electronics in Agriculture, vol. 828, (2012), pp. 7-95. 
Authors

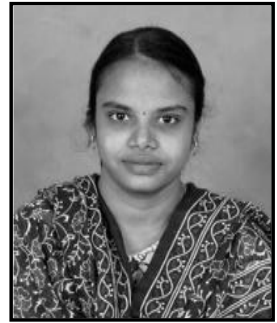

K. Lavanya, she is working as Assistant Professor in the school of computing science and engineering at VIT University, Vellore, Tamilnadu, India. Her area of interests includes Distributed Systems, Artificial Intelligence, High Performance computing and Intelligent computing. She is currently doing her research work in the interdisciplinary field of agriculture, computer science and tries to solve out the problems in agriculture farming practices such as marketing, crop management activities, disease control etc., using machine learning techniques.

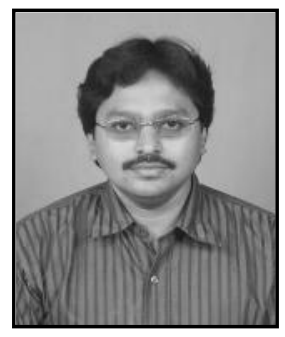

M. A. Saleem Durai, he received the MCA from Bharathidasan University,Tiruchirapalli, Tamilnadu, India in 1998; M. Phil. from from Madurai Kamaraj University, India in 2008 and completed his $\mathrm{PhD}$ at VIT University Vellore,India in 2011. He is an Associate Professor in the School of Computing Sciences and Engineering at VIT University, Vellore, Tamilnadu, India. He has authored many International and National journal papers to his credit. His research interests include data mining, fuzzy logic, cloud computing and rough sets. Mr. Saleem Durai is associated with many professional bodies CSI and IEEE.

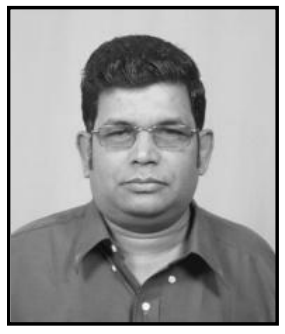

N. Ch. S. N. Iyengar, (1961) he is currently Senior Professor at the School of Computing Science and Engineering at VIT University, Vellore-632014, Tamil Nadu, India. His research interests include Agent-Based Distributed Secure Computing, Intelligent Computing, Network Security, Secured Cloud Computing and Fluid Dynamics. He authored several textbooks and had nearly 167 research publications in reputed peer reviewed international journals. He served as PCM/reviewer for many international conferences. He is Editor in Chief of International Journal of Software Engineering and Applications( IJSEA) of AIRCC, Guest Editor for Special Issue on Cloud Computing and Services of International Journal of Communications, Network and System Sciences and Editorial Board member for International Journals like IJConvC (Inderscience -China) etc. 